\title{
Summability and existence results for quasilinear parabolic equations with Hardy potential term
}

\author{
Maria Michaela Porzio and Ana Primo
}

\begin{abstract}
In this paper we study the existence and the summability of the solutions for a class of nonlinear parabolic equations with Hardy potential term. In particular we show how the presence of this singular potential and the summability of the datum $f$ influence the regularity of the solutions.
\end{abstract}

Mathematics Subject Classification (2000). 35K55, 35B65, 35R05.

Keywords. Nonlinear parabolic equations, Singular potentials, Regularity of the solutions.

\section{Introduction}

Let $\Omega$ be a bounded domain in $\mathbf{R}^{N}, N \geq 3$, containing the origin. For $T>0$, let us denote by $Q$ the cylinder $\Omega \times(0, T)$, and by $\Gamma$ the lateral surface $\partial \Omega \times(0, T)$. We will consider the following nonlinear parabolic Cauchy-Dirichlet problem

$$
\left\{\begin{aligned}
u_{t}-\operatorname{div}(a(x, t, u, \nabla u)) & =\lambda \frac{u}{|x|^{2}}+f \quad \text { in } Q, \\
u(x, t) & =0 \text { on } \Gamma, \\
u(x, 0) & =u_{0}(x) \text { in } \Omega,
\end{aligned}\right.
$$

where $\lambda$ is a positive constant and $f$ is a function in $L^{r}\left(0, T ; L^{q}(\Omega)\right), r \geq 1$, $q \geq 1$. Here $a(x, t, \sigma, \xi): Q \times \mathbf{R} \times \mathbf{R}^{N} \rightarrow \mathbf{R}^{N}$ is a Caratheodory function (i.e. it is continuous with respect to $(\sigma, \xi)$ for a.e. $(x, t) \in Q$, and measurable with respect to $(x, t)$ for every $\left.(\sigma, \xi) \in \mathbf{R} \times \mathbf{R}^{N}\right)$ satisfying

$$
\begin{gathered}
a(x, t, \sigma, \xi) \xi \geq \varrho_{1}|\xi|^{2}, \quad \varrho_{1}>0, \\
|a(x, t, \sigma, \xi)| \leq \varrho_{2}[|\xi|+|\sigma|+\mu(x, t)], \quad \varrho_{2}>0, \quad \mu \in L^{2}(Q), \\
\left(a(x, t, \sigma, \xi)-a\left(x, t, \sigma, \xi^{\prime}\right)\right) \cdot\left(\xi-\xi^{\prime}\right)>0, \quad \xi \neq \xi^{\prime} .
\end{gathered}
$$


This paper analyzes the existence and the regularity of the solutions of these nonlinear parabolic equations, emphasizing the action of the singular term $\frac{u}{|x|^{2}}$.

The model problem is the following

$$
\left\{\begin{aligned}
u_{t}-\Delta u & =\lambda \frac{u}{|x|^{2}}+f \quad \text { in } Q, \\
u(x, t) & =0 \text { on } \Gamma, \\
u(x, 0) & =u_{0}(x) \quad \text { in } \Omega .
\end{aligned}\right.
$$

In [7] it is proved that if the data $f$ and $u_{0}$ are nonnegative and not both identically zero (otherwise the result is false because $u \equiv 0$ is a solution) there is a dimension dependent constant $\Lambda_{N}$ such that problem (1.5) has no solution for $\lambda>\Lambda_{N}$. More in details, $\Lambda_{N}=\left(\frac{N-2}{2}\right)^{2}$ is the optimal constant in the Hardy's inequality

$$
\int_{\Omega}|\nabla \phi|^{2} d x \geq \Lambda_{N} \int_{\Omega} \frac{|\phi|^{2}}{|x|^{2}} d x, \quad \forall \phi \in \mathcal{C}_{0}^{\infty}(\Omega),
$$

and it is not attained (see for instance [18] and [15]).

Here, under the condition $\lambda<\Lambda_{N}$, that in the general case of problem (1.1) becomes $\lambda<\rho_{1} \Lambda_{N}$, we prove existence and regularity results for the general problem (1.1).

The presence of the singular potential has a strong influence on the regularity of the solutions. Even when the summability coefficients of the datum $f$ are sufficiently large, namely,

$$
\frac{1}{r}+\frac{N}{2 q}<1, \quad \text { (zone A in Fig. } 1 \text { below ) }
$$

differently from the classical case $\lambda=0$ (see [6]), we cannot expect bounded solutions because, as proved in [7], every solution of the model problem (1.5) (if the data $f$ and $u_{0}$ are nonnegative and not both identically zero) satisfies

$$
u(x, t) \geq \frac{C}{|x|^{\alpha_{1}}}, \quad(x, t) \in \Omega^{\prime} \times[\varepsilon, T),
$$

for every $\varepsilon \in(0, T)$ and $\Omega^{\prime} \subset \subset \Omega$, where the constant $C$ depends only on $\varepsilon$ and $\Omega^{\prime}$ and $\alpha_{1}$ is the smallest root of $\alpha^{2}-(N-2) \alpha+\lambda=0$. Notice that the previous result holds true for every choice of $0<\lambda<\Lambda_{N}$, and hence also for small value of $\lambda$.

It would be reasonable to expect that if the presence of this singular potential produces a lack of regularity when the datum is "good", i.e, so regular as we want, all the more reason there will be a lack of regularity also for a less regular datum.

Surprisingly it is not ever the case. Indeed what happens when (1.7) is not satisfied depends not only on how large are the coefficients of summability $r$ and $q$ but also, differently from the previous case, on the size of $\lambda$.

More in details, (see Theorem 1.3 below), if $\lambda$ is not too large, i.e. if $0<\lambda<\lambda_{*}$, where $\lambda_{*}$ can be explicitly determined in dependence on $N, q$, $r$ and the coercitivity constant $\rho_{1}$ (see Eq. (1.24)), for every choice of the 
summability exponents $r$ and $q$, also very small, we obtain the existence of a solution having the same regularity of the solutions of the same parabolic problem but with zero singular potential (i.e (1.1) with $\lambda=0$ ). More in details there exists a constant $\gamma_{*}>\frac{1}{2}$ (see Eq. (1.25)), that can be given explicitly in dependence on $r, q$ and $N$, and is the same value determined in [20] (see Theorem 9.1, chap. 3) and in [14] for $\lambda=0$, such that

$$
\left[(1+|u|)^{\gamma}-1\right] \operatorname{sign}(u) \in V^{2}(Q) \equiv L^{\infty}\left(0, T ; L^{2}(\Omega)\right) \cap L^{2}\left(0, T ; H_{0}^{1}(\Omega)\right),
$$

with $\gamma=\gamma_{*}$. Moreover, if the exponents $r$ and $q$ are sufficiently large, namely,

$$
\frac{N}{2 q}+\frac{1}{r} \leq 1+\frac{N}{4} \text { and } q \geq \frac{2 N}{N+2}, \quad q \in(1,+\infty), \quad r \in(1,+\infty),
$$

(zone 2, 3 and 4 of Fig. 2)

then $\gamma_{*}>1$ and hence $u \in V^{2}(Q)$.

Notice that the regularity (1.9) implies for example (see Remark 1.9 for further results) that

$$
u \in L^{2 \gamma_{*}}\left(0, T ; L^{2^{*} \gamma_{*}}(\Omega)\right), \quad \text { where } \quad 2^{*} \equiv \frac{2 N}{N-2} .
$$

The regularity $2^{*} \gamma_{*}$ in the space variable that we prove for $0<\lambda<\lambda_{*}$ seems to be sharp. As a matter of fact, in the case of the model problem (1.5), condition (1.8) implies, assuming for example that the ball $B_{1}$ of radius one and centered in the origin is contained in $\Omega$, that

$$
+\infty>\int_{\frac{T}{2}}^{T} \int_{B_{1}}|u(x, t)|^{2^{*} \gamma} \geq \frac{T}{2} \int_{B_{1}} \frac{C}{|x|^{\alpha_{1} 2^{*} \gamma}},
$$

and this happens if and only if

$$
\alpha_{1} 2^{*} \gamma<N
$$

For $\gamma=\gamma_{*},(1.12)$ becomes

$$
0<\lambda<\lambda_{*}
$$

If $\lambda$ is large, that is if $\lambda_{*} \leq \lambda<\rho_{1} \Lambda_{N}$ and $r$ and $q$ satisfy (1.10), we have again that $\left[(1+|u|)^{\gamma}-1\right] \operatorname{sign}(u) \in V^{2}(Q)$ with $\gamma>1$ but obviously $\gamma<\gamma_{*}$ depending now also on the size of $\lambda$ in a continuous and decreasing way.

Differently from the parabolic case, there is an extensive literature on elliptic problems with a Hardy potential (see for example $[2-5,13,17,23]$ ). However, it is not known if a similar phenomenon holds true in the stationary case, i.e., what happens to the regularity of the solutions for $\lambda$ not too small. Hence this argument will be treated in a forthcoming paper (see [22]).

Finally, we have thought in a different way to obtain further summability results (see Sect. 4). Although there aren't bounded solutions, it is difficult to believe that more summability properties of the datum $f$ don't imply more 
summability properties of the solution. It seems to be reasonable to hope that the case when $f \in L^{r}\left(0, T ; L^{q}(\Omega)\right)$, with $r$ and $q$ satisfying

$$
\frac{1}{r}+\frac{N}{2 q}<1, \quad \text { (zone A in Fig. } 1 \text { below ) }
$$

(that, as said before, in absence of a Hardy potential produces the boundedness of all the solutions belonging to the energy space $\left.V^{2}(Q)\right)$ produces more regularity respect to the case when $f$ is less regular.

For sake of simplicity, we have restricted this study to the case of the model problem (1.5) and we have proved that, as we expect, the regularity of the solutions increases with the regularity of the datum $f$. In particular, if the summability coefficients $r$ and $q$ satisfy (1.13) for every $0<\lambda<\Lambda_{N}$, there exists a solution $u$ of (1.5) satisfying

$$
u|x|^{\alpha_{1}} \in L^{\infty}(Q)
$$

where, as before, $\alpha_{1}$ is the smallest root of $\alpha^{2}-(N-2) \alpha+\lambda=0$, i.e.

$$
\alpha_{1}=\frac{N-2}{2}-\sqrt{\left(\frac{N-2}{2}\right)^{2}-\lambda} .
$$

We notice explicitly that the previous regularity result holds without any restriction on $\lambda$.

We denote $\alpha_{2}$ the other root, that is

$$
\alpha_{2}=\frac{N-2}{2}+\sqrt{\left(\frac{N-2}{2}\right)^{2}-\lambda} .
$$

Such roots appear in the radial solutions $|x|^{-\alpha_{1}},|x|^{-\alpha_{2}}$ to the equation

$$
-\Delta u-\lambda \frac{u}{|x|^{2}}=0
$$

Notice that $\alpha_{1}$ is an increasing and continuous function in $\lambda$; hence for $0 \leq$ $\lambda \leq \Lambda_{N}$, we have $\alpha_{1}(0)=0 \leq \alpha_{1}(\lambda) \leq \alpha_{1}\left(\Lambda_{N}\right)=\frac{N-2}{2}$.

Thus for $\lambda$ that tends to zero the regularity result (1.14) becomes the classical boundedness result of [6].

Moreover we have studied also the case when (1.13) is not satisfied and again we have obtained regularity results that hold true for every choice of $\lambda$, but that obviously are influenced by its size (for details see Sect. 4).

Let us represent the summability of the datum $f \in L^{r}\left(0, T ; L^{q}(\Omega)\right)$ in a diagram with axes $\frac{1}{q}$ and $\frac{1}{r}$. Since $r, q \in[1,+\infty]$, then all the possible cases of summability are inside of the square $[0,1] \times[0,1]$ (we use the notation $\left.\frac{1}{\infty}=0\right)$. We design two squares with different summability cases and that we will explain in next sections. 


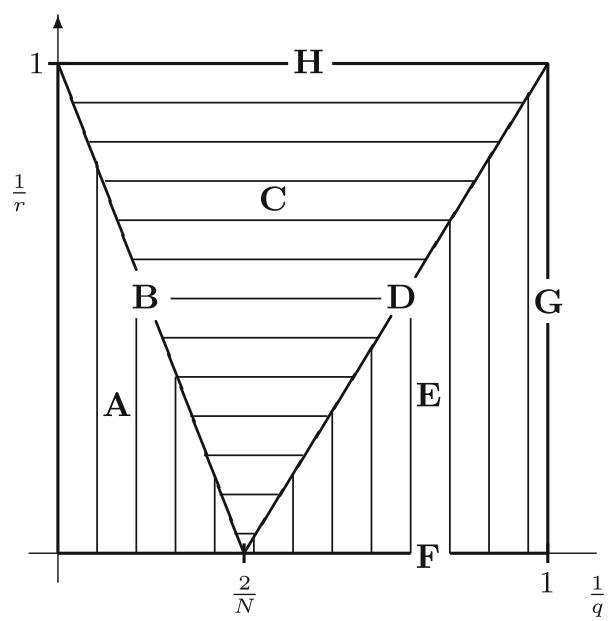

Figure 1. Summability cases

In Fig. 1 the zones $A, C$ and $E$ are defined by

Zone A: $\quad \frac{1}{r}+\frac{N}{2 q}<1$,

Zone C: $\quad \frac{N r}{N+2(r-1)} \leq q<\frac{N}{2} r^{\prime}, \quad$ where $\quad \frac{1}{r}+\frac{1}{r^{\prime}}=1$,

Zone E: $\quad 1<q<\frac{N r}{N+2(r-1)}$,

delimited by the lines

Line B: $\quad \frac{1}{r}+\frac{N}{2 q}=1, \quad$ Line D: $\quad q=\frac{N r}{N+2(r-1)}$,

Line F: $r=\infty, q>1, \quad$ Line G: $q=1, r>1, \quad$ Line $\mathrm{H}: \quad r=1, q>1$.

In Fig. 2, the zones 2 and 3 are separated by the line $r=2$ while the zones 2 and 6 are separated by the line $\frac{N}{2 q}+\frac{1}{r}=1+\frac{N}{4}$. Notice that the zona $C$ in Fig. 1, can be obtained by the union of the zone 2, 3 and 6 in the Fig. 2, while the zone $E$ in Fig. 1 can be obtain by putting together the line $q=\frac{2 N}{N+2}$ and the zones 4 and 5 in Fig. 2.

Now we enounce our results whose proofs can be found in the following sections.

\subsection{Statement of the results}

Before stating our results we recall the definition of a solution to (1.1).

Definition 1.1. We say that $u \in L^{\infty}\left(0, T ; L^{1}(\Omega)\right) \cap L^{1}\left(0, T ; W_{0}^{1,1}(\Omega)\right)$ is a weak supersolution (subsolution) to problem (1.1), if $a(x, t, u, \nabla u) \in L^{1}(Q), \frac{|u|}{|x|^{2}} \in$ $L^{1}(Q), f \in L^{1}(Q)$ and for all $\phi \in C^{\infty}(\bar{Q})$ such that $\phi=0$ in a neighborhood 


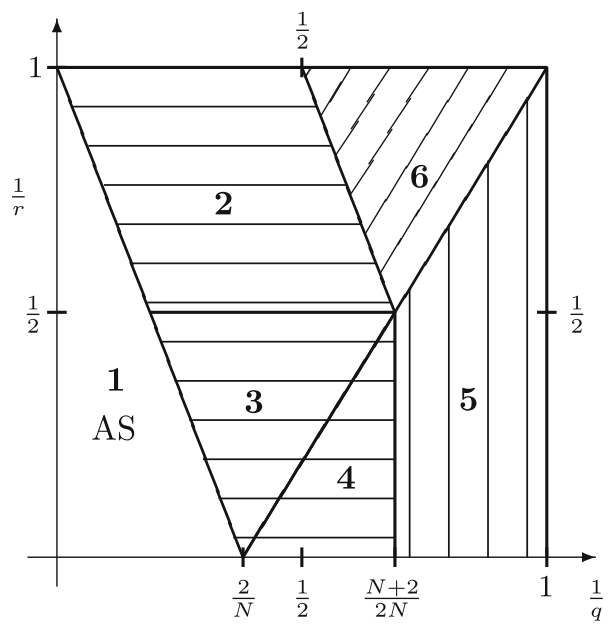

FiguRE 2. Summability cases

of $\Gamma \cup\{\Omega \times\{T\}\}$ and $\phi \geq 0$ we have

$$
\begin{aligned}
& \int_{0}^{T} \int_{\Omega}\left\{-\phi_{t} u+a(x, t, u, \nabla u) \nabla \phi\right\} d x d t \geq(\leq) \\
& \int_{0}^{T} \int_{\Omega}\left(\lambda \frac{u}{|x|^{2}}+f\right) \phi d x d t+\int_{\Omega} u_{0} \phi(x, 0) d x .
\end{aligned}
$$

If $u$ is a super and sub weak solution, then we say that $u$ is a weak solution.

We start studying the following case

$$
\frac{N}{2 q}+\frac{1}{r} \leq 1+\frac{N}{4} \text { and } q \geq \frac{2 N}{N+2}, \quad q \in(1,+\infty), \quad r \in(1,+\infty) .
$$

Notice that in this case the values $\left(\frac{1}{r}, \frac{1}{q}\right)$ belong to the zones $1,2,3,4$ of Fig. 2 and in absence of a singular potential this regularity of the datum $f$ guarantees the existence of a solution in the energy space $V^{2}(Q)$. The following result states that the same also happens in presence of a Hardy potential. More in details we have.

Theorem 1.2. Assume (1.2)-(1.4). If $f \in L^{r}\left(0, T ; L^{q}(\Omega)\right)$ satisfies (1.19), then there exists a solution $u \in V^{2}(Q)$ to problem (1.1), for all $\lambda<\varrho_{1} \Lambda_{N}$ and $u_{0} \in L^{2}(\Omega)$, where $\varrho_{1}$ is the coercivity constant defined in (1.2) and $\Lambda_{N}$ is the optimal Hardy constant defined in (1.6).

Indeed, as just said in the introduction, it is possible to prove further regularity results when (1.19) holds true. Moreover it is possible to prove the existence (and the regularity) of solutions also when (1.19) is not satisfied and $\lambda$ is not too large. We state these results all together in the following theorem. 
Theorem 1.3. Let us assume (1.2)-(1.4), $f \in L^{r}\left(0, T ; L^{q}(\Omega)\right)$ where $r \in(1,+\infty)$, $q \in(1,+\infty)$ and $u_{0} \in L^{\infty}(\Omega)$. If

$$
\frac{1}{r}+\frac{N}{2 q} \leq 1, \quad \text { (zone } A \text { in Fig. 1) }
$$

then for every $0<\lambda<\varrho_{1} \Lambda_{N}$ there exists a weak solution $u$ of (1.1) satisfying

$$
\begin{aligned}
& {\left[(|u|+1)^{\gamma}-1\right] \operatorname{sign}(u) \in V^{2}(Q) \cap L^{p}\left(0, T ; L^{s}(\Omega)\right), \quad p=\frac{4 \gamma m}{N(m-2)},} \\
& \quad s=\gamma m, \quad \forall m \in\left[2,2^{*}\right],
\end{aligned}
$$

for every $\gamma<\gamma^{*}$, where $\gamma^{*}>1$ is defined as

$$
\gamma^{*}=\gamma^{*}(\lambda)=\frac{\Lambda_{N} \varrho_{1}+\sqrt{\Lambda_{N} \varrho_{1}\left(\Lambda_{N} \varrho_{1}-\lambda\right)}}{\lambda} .
$$

If otherwise

$$
\left.\frac{1}{r}+\frac{N}{2 q}>1, \quad \text { (zones } C \text { and } E \text { in Fig. } 1\right)
$$

there exists a constant $\lambda_{*} \in\left(0, \varrho_{1} \Lambda_{N}\right)$ given by the following formula

$$
\lambda_{*}= \begin{cases}\lambda_{1}=\varrho_{1} \frac{N(q-1)(N-2 q)}{2 q^{2}}=\frac{\varrho_{1} N^{2}}{q^{\prime} q^{* *}}, \quad \text { if } 1<q<\frac{N r}{N+2(r-1)}, \\ \lambda_{2}=\varrho_{1}(N-2)^{2}\left(\frac{1}{q}-\frac{2}{r^{\prime} N}\right)\left(\frac{1}{q^{\prime}}+\frac{2}{r^{\prime} N}\right), \text { if } \frac{N r}{N+2(r-1)} \leq q<\frac{N}{2} r^{\prime},\end{cases}
$$

such that for every $0<\lambda<\lambda_{*}$ there exists a weak solution $u$ of (1.1) satisfying (1.21) with $\gamma=\gamma_{*}$, where $\gamma_{*}>\frac{1}{2}$ is defined as follows

$$
\gamma_{*}=\left\{\begin{array}{l}
\gamma_{2}=\frac{1}{2} \frac{q r N}{N r-2 q(r-1)} \quad \text { if } \frac{N r}{N+2(r-1)} \leq q<\frac{N}{2} r^{\prime},(\text { zone C in Fig. } 1), \\
\gamma_{1}=\frac{1}{2} \frac{q(N-2)}{N-2 q}=\frac{q^{* *}}{2^{*}} \text { if } 1<q<\frac{N r}{N+2(r-1)}, \quad \text { (zone E in Fig. 1). }
\end{array}\right.
$$

Finally if we assume $\lambda_{*} \leq \lambda<\varrho_{1} \Lambda_{N}$ and

$$
1<\frac{N}{2 q}+\frac{1}{r} \leq 1+\frac{N}{4} \text { and } q \geq \frac{2 N}{N+2}, \quad \text { (zones 2, } 3 \text { and } 4 \text { in Fig. 2) }
$$

there exists a weak solution $u$ of (1.1) satisfying (1.21) for every $\gamma<\gamma^{*}$, where $\gamma^{*}>1$ is as in (1.22).

Here we have used the following notations

$$
q^{*}=\frac{q N}{N-q}, \quad q^{* *}=\left(q^{*}\right)^{*}=\frac{q N}{N-2 q}, \quad \text { and } \quad \frac{1}{r}+\frac{1}{r^{\prime}}=1 .
$$

Remark 1.4. Notice that when $\lambda$ is not too large, that is if $0<\lambda<\lambda_{*}$, and (1.23) holds true, the Hardy potential doesn't influence the regularity of the solution we have found. As a matter of fact, the regularity (1.21) with $\gamma=\gamma_{*}$ is the same regularity proved in absence of the Hardy potential (see [14]). 
On the contrary, in all the other cases, i.e. if (1.23) and $\lambda_{*} \leq \lambda<\varrho_{1} \Lambda_{N}$ hold or if (1.20) is satisfied, the Hardy potential influence the regularity of $u$.

Remark 1.5. Observe that $\gamma^{*}$ tends to $+\infty$ for $\lambda$ that tends to zero. Hence if (1.20) holds true the influence of the Hardy potential decreases with $\lambda$ and for $\lambda$ which goes to zero we get the classical boundedness result of [6].

Remark 1.6. Notice that it results $\gamma_{*} \geq 1$ if and only if (1.26) holds true. Moreover if (1.26) is assumed, for $\lambda \rightarrow \lambda_{*}$ we get $\gamma_{*} \rightarrow \gamma^{*}$; hence if (1.26) holds the exponent $\gamma$ is a continuous function of $\lambda$ in all the interval $\left(0, \varrho_{1} \Lambda_{N}\right)$. Finally if $\lambda_{*}<\lambda<\rho_{1} \Lambda_{N}$ and (1.26) hold true it results $\gamma^{*}<\gamma_{*}$ (since $\gamma^{*}(\lambda)$ is a decreasing function of $\lambda$ ) and hence also in this case the Hardy potential produces a reduction of regularity which decrease (in a continuous way) with $\lambda$.

Remark 1.7. Notice that the condition $\gamma<\gamma^{*}$ (with $\gamma^{*}$ as in (1.22)) seems sharp since when $\varrho_{1}=1$ it becomes (1.12).

Remark 1.8. For sake of simplicity we have assumed $u_{0} \in L^{\infty}(\Omega)$ but the result of Theorem 1.3 holds if $u_{0} \in L^{2 \gamma}(\Omega)$, where $\gamma$ is as above.

Remark 1.9. Notice that choosing $m=2^{*}$ in (1.21), we get the best summability exponent in the spacial variable (i.e. $2^{*} \gamma$ ) together with the lower summability exponent in the time variable (i.e. $2 \gamma$ ) that is

$$
u \in L^{2 \gamma}\left(0, T ; L^{2^{*} \gamma}(\Omega)\right) .
$$

In particular if $0<\lambda<\lambda_{*}$ and

$$
\text { if } 1<q<\frac{N r}{N+2(r-1)}, \quad \text { (area E in the Fig. 1); }
$$

we have that (1.27) holds with

$$
2 \gamma=2 \gamma_{1}=p_{1}=\frac{2}{2^{*}} q^{* *}=\frac{q(N-2)}{(N-2 q)}>q, \quad 2^{*} \gamma=2^{*} \gamma_{1}=s_{1} \equiv q^{* *},
$$

while if $0<\lambda<\lambda_{*}$ and

$$
\text { if } \frac{N r}{N+2(r-1)} \leq q<\frac{N}{2} r^{\prime}, \quad \text { (zone C in Fig. 1) }
$$

(1.27) holds with

$$
2 \gamma=2 \gamma_{2}=\frac{q r N}{N r-2 q(r-1)}, \quad 2^{*} \gamma=2^{*} \gamma_{2}=\frac{q r N^{2}}{[N r-2 q(r-1)]) N-2)} .
$$

Finally (if $0<\lambda<\lambda_{*}$ ), choosing $m=2 \frac{N+2}{N}$ in (1.21), we get $p=s$ and hence

$$
u \in L^{\bar{s}}(Q), \quad \bar{s}=2 \gamma \frac{N+2}{N},
$$

that is

$$
\bar{s}=\overline{s_{1}} \equiv \frac{q\left(N^{2}-4\right)}{N(N-2 q)}
$$

if (1.28) holds. 
Otherwise (i.e. if (1.29) holds) we have

$$
u \in L^{\bar{s}}(Q), \quad \bar{s}=\overline{s_{2}} \equiv \frac{q r(N+2)}{N r+2 q-2 q r} .
$$

Moreover in this last case (zone C in Fig. 1), choosing $m=2+4 \frac{q^{\prime}}{r^{\prime} N}$, we get

$$
u \in L^{p_{2}}\left(0, T ; L^{s_{2}}(\Omega)\right), \quad p_{2}=\rho r^{\prime}, \quad s_{2}=\rho q^{\prime}, \quad \rho=\frac{r N(q-1)+2 q(r-1)}{r(N-2 q)+2 q} .
$$

Remark 1.10. We observe that summability results for $D u$ are proved in the proof of Theorem 1.3. In particular, if (1.23) is satisfied but (1.26) does not hold (i.e. in zones 5 and 6 of Fig. 2) we have that

$$
D u \in\left(L^{M}(Q)\right)^{N}, \quad \forall 0<\lambda<\lambda_{*},
$$

where $M>1$ is defined by $M=1+\sqrt{1-\frac{N\left(1-\gamma_{*}\right)}{\gamma_{*}(N+2)}}$, with $\gamma_{*}$ as in (1.25) (for details, see Remark 3.2).

In the following section we give some preliminary results while in Sect. 3 we prove the previous results. Finally, further regularity results can be found in Sect. 4.

\section{Preliminary results}

We recall some embedding properties we will use in the following.

Lemma 2.1. Let $v$ be a function in $W_{0}^{1, h}(\Omega) \cap L^{\rho}(\Omega)$, with $h \geq 1$ and $\rho \geq 1$. Then there exists a positive constant $C_{1}$, depending only on $N, h$ and $\rho$, such that

$$
\|v\|_{L^{\eta}(\Omega)} \leq C_{1}\|\nabla v\|_{L^{h}(\Omega)}^{\theta}\|v\|_{L^{\rho}(\Omega)}^{1-\theta},
$$

for every $\eta$ and $\theta$ satisfying

$$
0 \leq \theta \leq 1, \quad 1 \leq \eta<+\infty, \quad \frac{1}{\eta}=\theta\left(\frac{1}{h}-\frac{1}{N}\right)+\frac{1-\theta}{\rho} .
$$

Proof. See [21], Lecture II.

A first and immediate consequence of the previous lemma is the following embedding result

$$
\int_{Q}|v|^{\sigma} \leq C\left[\sup _{[0, T]} \int_{\Omega}|u|^{\rho} d x\right]^{\frac{h}{N}} \int_{Q}|\nabla v|^{h}
$$

which holds for every function $v \in L^{h}\left(0, T ; W_{0}^{1, h}(\Omega)\right) \cap L^{\infty}\left(0, T ; L^{\rho}(\Omega)\right)$, with $h \geq 1, \rho \geq 1$ and $\sigma=\frac{h(N+\rho)}{N}$ (see [17], Proposition 3.1).

For $h=2$, we get the following result. 
Lemma 2.2. Let $v$ be a function in $L^{2}\left(0, T ; H_{0}^{1}(\Omega)\right) \cap L^{\infty}\left(0, T ; L^{\rho}(\Omega)\right)$, with $\rho \geq 1$. Then $v$ belongs to $L^{m}(Q)$, with $m=2 \frac{N+\rho}{N}$.

A second consequence of (2.1) is the following immersion property.

Lemma 2.3. Every function $v$ belonging to $V^{2}(Q)$ belongs also to $L^{\delta}(0, T$; $\left.L^{\eta}(\Omega)\right)$ for every $\eta \in\left[2,2^{*}\right]$ and $\delta=\frac{4 \eta}{N(\eta-2)}$, where we use the notation $\frac{1}{0}=+\infty$.

Proof. Consider inequality (2.1) with $h=\rho=2$. Thus it results $\frac{1}{\eta}=\frac{1}{2}-\frac{\theta}{N}$ for every fixed $\theta \in[0,1]$. Elevating to the power $\delta=\frac{2}{\theta}=\frac{4 \eta}{N(\eta-2)}$ and integrating in the time variable we deduce

$$
\begin{aligned}
\left(\int_{0}^{T}\|v\|_{L^{\eta}(\Omega)}^{\delta} d t\right)^{\frac{1}{\delta}} & \leq\left[C \int_{0}^{T}\|\nabla v\|_{2}^{\theta \delta}\|v\|_{2}^{(1-\theta) \delta}\right]^{\frac{1}{\delta}} \\
& \leq C^{\frac{1}{\delta}}\|v\|_{L^{\infty}\left(0, T ; L^{2}(\Omega)\right)}^{1-\theta}\left[\int_{0}^{T}\|\nabla v\|_{2}^{\theta \delta} d t\right]^{\frac{1}{\delta}},
\end{aligned}
$$

from which the assert follows recalling the definition of $\delta$.

Let $s \in \mathbf{R}$ and denote $L_{s}^{r}(\Omega)$ the following set,

$$
L_{s}^{r}(\Omega)=\left\{f \text { measurable, such that } \int_{\Omega}|x|^{-r s}|f|^{r} d x<\infty\right\} .
$$

Moreover, we define $W_{0, s}^{1, r}(\Omega)$ as the closure of $C_{c}^{\infty}(\Omega)$ with the norm

$$
\|f\|_{L_{s}^{r}(\Omega)}^{r}=\int_{\Omega}|x|^{-r s}|\nabla f|^{r} d x<\infty
$$

and we denote $V_{\alpha_{1}}^{2}(Q) \equiv L^{\infty}\left(0, T ; L_{\alpha_{1}}^{2}(\Omega)\right) \cap L^{2}\left(0, T ; W_{0, \alpha_{1}}^{1,2}(\Omega)\right)$. We have the following immersion properties.

Proposition 2.4 (Caffarelli-Kohn-Nirenberg). Let be $N \geq 3$ and suppose that $p, q, r, s, k, \sigma$ and a are real constants and $p, q \geq 1, r>0,0 \leq \theta \leq 1$ satisfying

$$
\frac{1}{p}+\frac{s}{N}>0 \quad \text { and } \quad \frac{1}{q}+\frac{k}{N}>0 .
$$

Denote $m=\theta \sigma+(1-\theta) k$. There exists a positive constant $C$ such that for every $u \in \mathcal{C}_{0}^{\infty}\left(\mathbb{R}^{N}\right)$ we have that

$$
\left\||x|^{m} u\right\|_{L^{r}\left(\mathbb{R}^{N}\right)} \leq C\left\||x|^{s}|\nabla u|\right\|_{L^{p}\left(\mathbb{R}^{N}\right)}^{\theta}\left\||x|^{k} u\right\|_{L^{q}\left(\mathbb{R}^{N}\right)}^{1-\theta},
$$

if and only if the following equalities hold

$$
\begin{gathered}
\frac{1}{r}+\frac{m}{N}=\theta\left(\frac{1}{p}+\frac{s-1}{N}\right)+(1-\theta)\left(\frac{1}{q}+\frac{k}{N}\right), \\
0 \leq s-\sigma \text { if } \theta>0,
\end{gathered}
$$

and

$$
s-\sigma \leq 1 \quad \text { if } \quad \theta>0 \quad \text { and } \quad \frac{1}{r}+\frac{m}{N}=\frac{1}{p}+\frac{s-1}{N}
$$


Notice that by density arguments the previous result holds with $\mathbb{R}^{N}$ replaced by $\Omega$ in $(2.2)$ if $u \in L_{-k}^{q}(\Omega) \cap W_{0,-s}^{p}(\Omega)$. A consequence of Proposition 2.4 is the following immersion property.

Lemma 2.5. Every function $v$ belonging to $V_{\alpha_{1}}^{2}(Q)$ belongs also to $L^{\delta}(0, T$; $\left.L_{\alpha_{1}}^{\eta}(\Omega)\right)$ for every $\eta \in\left[2,2^{*}\right]$ and $\delta=\frac{4 \eta}{N(\eta-2)}$.

Proof. Consider inequality (2.3) with $p=q=2, m=s=k=-\alpha_{1}$ and $r=\eta$. Thus it results $\frac{1}{\eta}=\frac{1}{2}-\frac{\theta}{N}$ for every fixed $\theta \in[0,1]$. Elevating $(2.2)$ (written in $\Omega$ ) to the power $\delta=\frac{2}{\theta}=\frac{4 \eta}{N(\eta-2)}$ and integrating in the time variable we deduce

$$
\begin{aligned}
\left(\int_{0}^{T}\left\||x|^{-\alpha_{1}} v\right\|_{L^{\eta}(\Omega)}^{\delta} d t\right)^{\frac{1}{\delta}} & \leq\left[C \int_{0}^{T}\left\||x|^{-\alpha_{1}} \nabla v\right\|_{2}^{\theta \delta}\left\||x|^{-\alpha_{1}} v\right\|_{2}^{(1-\theta) \delta}\right]^{\frac{1}{\delta}} \\
& \leq C^{\frac{1}{\delta}}\|v\|_{L^{\infty}\left(0, T ; L_{\alpha_{1}}^{2}(\Omega)\right)}^{1-\theta}\left[\int_{0}^{T}\left\||x|^{-\alpha_{1}} \nabla v\right\|_{2}^{\theta \delta} d t\right]^{\frac{1}{\delta}},
\end{aligned}
$$

from which the assert follows recalling the definition of $\delta$.

As a consequence of the Caffarelli-Kohn-Nirenberg inequalities in Proposition 2.4, we have also the following Sobolev's weight inequality.

Theorem 2.6 (Sobolev's weight inequality). Let $u \in W_{0, \gamma}^{1,2}(\Omega)$ with $\gamma<\frac{N-2}{2}$. Then there exists a positive constant $C_{S o b}=C(N, \gamma)$ such that

$$
\left(\int_{\Omega}|u|^{2^{*}}|x|^{-2^{*} \gamma} d x\right)^{1 / 2^{*}} \leq C_{S o b}\left(\int_{\Omega}|\nabla u|^{2}|x|^{-2 \gamma} d x\right)^{1 / 2},
$$

where $2^{*}=\frac{2 N}{N-2}$. Notice that for $\gamma=0$, we have the classical Sobolev inequality

$$
\left(\int_{\Omega}|u|^{2^{*}} d x\right)^{1 / 2^{*}} \leq C_{S}\left(\int_{\Omega}|\nabla u|^{2} d x\right)^{1 / 2} .
$$

We recall a very well known lemma, useful to handle $L^{\infty}$ estimates, proved by Stampacchia in [25].

Lemma 2.7. Let us suppose that $\varphi$ is a real, non negative and non increasing function verifying

$$
\varphi(h) \leq \frac{C}{(h-k)^{\delta}}[\varphi(k)]^{\nu} \quad \forall h>k>k_{0},
$$

where $C$ and $\delta$ are positive constants and $\nu>1$. Then there exists a positive constant $d$ such that

$$
\varphi\left(k_{0}+d\right)=0 .
$$

We conclude with the classical compactness result of Simon. 
Lemma 2.8 (cf. [24], Corollary 4, p. 85). Let $X, B$, and $Y$ be Banach spaces such that

$$
X \subset B \subset Y,
$$

with compact imbedding $X \rightarrow B$. Let $F$ be bounded in $L^{q}(0, T ; X)$ where $1 \leq$ $q<\infty$, and $\frac{\partial F}{\partial t}=\{\partial f / \partial t: f \in F\}$ be bounded in $L^{1}(0, T ; Y)$. Then $F$ is relatively compact in $L^{q}(0, T ; B)$.

We notice explicitly that in all the paper the use of the test functions can be made rigorous by means of Steklov averaging process (see for example chapter 1 of [17]).

\section{Proof of Theorems 1.2 and 1.3}

\subsection{Proof of Theorem 1.2}

For every $n \in \mathbf{N}$ let us consider $u_{n} \in V^{2}(Q) \cap L^{\infty}(Q)$ the solution to the approximated problem

$$
\left\{\begin{array}{l}
\left(u_{n}\right)_{t}-\operatorname{div}\left(a\left(x, t, u_{n}, \nabla u_{n}\right)\right)=\lambda \frac{u_{n}}{|x|^{2}+\frac{1}{n}}+T_{n}(f) \quad \text { in } Q \\
u_{n}(x, t)=0 \quad \text { on } \partial \Omega \times(0, T) \\
u_{n}(x, 0)=u_{0}(x) \quad \text { in } \Omega
\end{array}\right.
$$

where $T_{n}$ is the truncation function, i.e.

$$
T_{n}(s)= \begin{cases}s, & \text { if }|s| \leq n \\ \frac{s}{|s|} n, & \text { if }|s|>n .\end{cases}
$$

We prove now the following estimate

$$
\left\|u_{n}\right\|_{L^{\infty}\left(0, T ; L^{2}(\Omega)\right)}+\left\|\nabla u_{n}\right\|_{L^{2}(Q)} \leq c_{0}
$$

where $c_{0}$ is a constant independent on $n$.

Take $u_{n}$ as a test function in (3.1). For every $t \in(0, T)$ we have

$$
\begin{aligned}
& \frac{1}{2} \int_{\Omega}\left|u_{n}\right|^{2}(t) d x+\varrho_{1} \int_{0}^{t} \int_{\Omega}\left|\nabla u_{n}\right|^{2} d x d \tau \\
& \quad \leq \lambda \int_{0}^{t} \int_{\Omega} \frac{\left|u_{n}\right|^{2}}{|x|^{2}} d x d \tau+\int_{0}^{t} \int_{\Omega} f u_{n} d x d \tau+\frac{1}{2} \int_{\Omega}\left|u_{0}(x)\right|^{2} d x .
\end{aligned}
$$

Let us estimate the first term on the right hand of (3.4). Since $\lambda<\varrho_{1} \Lambda_{N}$, there exists $\kappa_{0} \in\left(0, \varrho_{1}\right)$ such that $\lambda=\left(\varrho_{1}-\kappa_{0}\right) \Lambda_{N}$. Thus applying Hardy's inequality we have

$$
\lambda \int_{0}^{t} \int_{\Omega} \frac{\left|u_{n}\right|^{2}}{|x|^{2}} d x d \tau \leq\left(\varrho_{1}-\kappa_{0}\right) \int_{0}^{t} \int_{\Omega}\left|\nabla u_{n}\right|^{2} d x d \tau
$$

that with (3.4) gives

$$
\frac{1}{2} \int_{\Omega}\left|u_{n}\right|^{2}(t) d x+\kappa_{0} \int_{0}^{t} \int_{\Omega}\left|\nabla u_{n}\right|^{2} d x d \tau \leq \int_{0}^{t} \int_{\Omega} f u_{n} d x d \tau+\frac{1}{2} \int_{\Omega}\left|u_{0}(x)\right|^{2} d x .
$$


If $f \in L^{r}\left(0, T ; L^{q}(\Omega)\right)$ with $q^{\prime} \leq 2$ we have

$$
\begin{aligned}
& \int_{0}^{t} \int_{\Omega} f u_{n} d x d \tau \leq\left(\int_{0}^{t}\left(\int_{\Omega}|f|^{q} d x\right)^{\frac{r}{q}} d \tau\right)^{\frac{1}{r}}\left(\int_{0}^{t}\left(\int_{\Omega}\left|u_{n}\right|^{q^{\prime}} d x\right)^{\frac{r^{\prime}}{q^{\prime}}} d \tau\right)^{\frac{1}{r^{\prime}}} \\
& \quad \leq c_{1}\left(\int_{0}^{t}\left(\int_{\Omega}\left|u_{n}\right|^{2} d x\right)^{\frac{r^{\prime}}{2}}|\Omega|^{1-\frac{q^{\prime}}{2}} d \tau\right)^{\frac{1}{r^{\prime}}} \leq c_{1}|\Omega|^{\left(1-\frac{q^{\prime}}{2}\right) \frac{1}{r^{\prime}}}\left\|u_{n}\right\|_{L^{\infty}\left(0, T ; L^{2}(\Omega)\right)} \\
& \quad \leq \frac{1}{4}\left\|u_{n}\right\|_{L^{\infty}\left(0, T ; L^{2}(\Omega)\right)}^{2}+2 c_{1}^{2}|\Omega|^{\frac{2-q^{\prime}}{r^{\prime}}}
\end{aligned}
$$

where $c_{1}=\|f\|_{L^{r}\left(0, T ; L^{q}(\Omega)\right)}$. By (3.6) and (3.5) it follows (3.3).

If $f \in L^{r}\left(0, T ; L^{q}(\Omega)\right)$, with $q^{\prime}>2$ and $r \geq 2$, that is $r^{\prime} \leq 2$ and $2<q^{\prime} \leq 2^{*}$ (as we are assuming $q \geq \frac{2 N}{N+2}$ ), applying Hölder's inequality we get

$$
\begin{aligned}
& \int_{0}^{t} \int_{\Omega} f u_{n} d x d \tau \leq\left(\int_{0}^{t}\left(\int_{\Omega}|f|^{q} d x\right)^{\frac{r}{q}} d \tau\right)^{\frac{1}{r}}\left(\int_{0}^{t}\left(\int_{\Omega}\left|u_{n}\right|^{q^{\prime}} d x\right)^{\frac{r^{\prime}}{q^{\prime}}} d \tau\right)^{\frac{1}{r^{\prime}}} \\
& \leq c_{1}\left(\int_{0}^{t}\left(\int_{\Omega}\left|u_{n}\right|^{2^{*}} d x\right)^{\frac{r^{\prime}}{2^{*}}} d \tau\right)^{\frac{1}{r^{\prime}}} \leq c_{1} T^{1-\frac{r^{\prime}}{2}}\left(\int_{0}^{t}\left(\int_{\Omega}\left|u_{n}\right|^{2^{*}} d x\right)^{\frac{2}{2^{*}}} d \tau\right)^{\frac{1}{2}} \\
& \varepsilon\left\|u_{n}\right\|_{L^{2}\left(0, T ; L^{2 *}(\Omega)\right)}^{2}+c_{2},
\end{aligned}
$$

where $c_{1}$ is as before, $c_{2}=c_{1}^{2} \frac{T^{2-r^{\prime}}}{\varepsilon}$ and $\varepsilon=\frac{\kappa_{0}}{2} C_{S}^{2}$, with $C_{S}$ the Sobolev constant in (2.5). From (3.5) and (3.7), we get

$$
\frac{1}{2} \int_{\Omega}\left|u_{n}\right|^{2}(t) d x+\frac{\kappa_{0}}{2} \int_{0}^{t} \int_{\Omega}\left|\nabla u_{n}\right|^{2} d x d \tau \leq c_{2}+\frac{1}{2} \int_{\Omega}\left|u_{0}(x)\right|^{2} d x,
$$

and hence (3.3) holds true.

Finally, if $f \in L^{r}\left(0, T ; L^{q}(\Omega)\right)$ with $r<2$ and $q^{\prime}>2$ using the Interpolation's inequalities with $\theta \in(0,1), \frac{1}{q^{\prime}}=\frac{\theta}{2^{*}}+\frac{(1-\theta)}{2}$, observing that assumption (1.19) is equivalent to require $r^{\prime} \theta \leq 2$ and by means of Young inequality, we have

$$
\begin{aligned}
& \int_{0}^{t} \int_{\Omega} f u_{n} d x d \tau \leq\left(\int_{0}^{T}\left(\int_{\Omega}|f|^{q} d x\right)^{\frac{r}{q}} d \tau\right)^{\frac{1}{r}}\left(\int_{0}^{t}\left(\int_{\Omega}\left|u_{n}\right|^{q^{\prime}} d x\right)^{\frac{r^{\prime}}{q^{\prime}}} d \tau\right)^{\frac{1}{r^{\prime}}} \\
& \leq c_{1}\left(\int_{0}^{t}\left\|u_{n}\right\|_{L^{2}(\Omega)}^{\theta r^{\prime}}\left\|u_{n}\right\|_{L^{2}(\Omega)}^{(1-\theta) r^{\prime}} d \tau\right)^{\frac{1}{r^{\prime}}} \\
& \leq c_{1}\left[\left(\int_{0}^{t}\left\|u_{n}\right\|_{L^{2 *}(\Omega)}^{2}\right)^{\frac{\theta r^{\prime}}{2}}\left(\int_{0}^{t}\left\|u_{n}\right\|_{L^{2}(\Omega)}^{(1-\theta) r^{\prime}\left(\frac{2}{\theta r^{\prime}}\right)^{\prime}} d \tau\right)^{1-\frac{\theta r^{\prime}}{2}}\right]^{\frac{1}{r^{\prime}}} \\
& \leq c_{1}\left(\frac{1}{C_{S}^{2}} \int_{0}^{t} \int_{\Omega}\left|\nabla u_{n}\right|^{2}\right)^{\frac{\theta}{2}}\left\|u_{n}\right\|_{L^{\infty}\left(0, T ; L^{2}(\Omega)\right)}^{1-\theta} T^{\left(1-\frac{\theta r^{\prime}}{2}\right) \frac{1}{r^{\prime}}}
\end{aligned}
$$




$$
\begin{aligned}
& \leq \varepsilon_{1}\left\|\nabla u_{n}\right\|_{L^{2}(\Omega)}^{2}+c_{3}\left\|u_{n}\right\|_{L^{\infty}\left(0, T ; L^{2}(\Omega)\right)}^{\frac{2(1-\theta)}{2-\theta}} T^{\left(\frac{1}{r^{\prime}}-\frac{\theta}{2}\right)^{\frac{2}{2-\theta}}} \\
& \leq \varepsilon_{1}\left\|\nabla u_{n}\right\|_{L^{2}(\Omega)}^{2}+\varepsilon_{2}\left\|u_{n}\right\|_{L^{\infty}\left(0, T ; L^{2}(\Omega)\right)}^{2}+c_{4},
\end{aligned}
$$

where $c_{3}$ is a constant depending only on $\varepsilon_{1}, c_{1}$ and $C_{S}^{\theta}, \varepsilon_{1}=\frac{\kappa_{0}}{2}, \varepsilon_{2}=\frac{1}{4}$ and $c_{4}=c\left(\kappa_{0}, f, C_{S}, T, r, \theta\right)$. From (3.9) and (3.5) it follows that (3.3) holds true. By (3.3), the assert follows by means of standard converging arguments (see also the proof of Theorem 1.3).

\subsection{Proof of Theorem 1.3}

Let $u_{n}$ be as in (3.1). The proof proceeds in two steps. In the first one we prove that the regularity property (1.21) is uniformly satisfied (in $\mathrm{n}$ ) by $u_{n}$. In the second step we will conclude the proof passing to the limit in the approximating solutions $u_{n}$ and showing that the limit $\mathrm{u}$ satisfies the assertions of the Theorem.

First step. As noticed above, we prove here that there exists a constant C, independent of $n$, such that

$$
\begin{aligned}
& \left\|\left[\left(\left|u_{n}\right|+1\right)^{\gamma}-1\right] \operatorname{sign}\left(u_{n}\right)\right\|_{V^{2}(Q) \cap L^{p}\left(0, T ; L^{s}(\Omega)\right)} \leq C, \quad p=\frac{4 \gamma m}{N(m-2)}, \\
& s=\gamma m, \quad \forall m \in\left[2,2^{*}\right],
\end{aligned}
$$

where $\gamma$ is as in the statement of the Theorem 1.3 an exponent that depends on $\lambda$ and on the regularity exponents $r$ and $q$ of $f$ (thus all the different cases considered in Theorem 1.3 will be studied here).

To this aim, define for $s \in \mathbf{R}$

$$
\Psi(s)=\int_{0}^{s}\left[(1+|y|)^{2 \gamma-1}-1\right] \operatorname{sign}(y) d y,
$$

where $\gamma$ is a positive constant verifying $\gamma>\frac{1}{2}$ that will be chosen later as in the statement of the Theorem 1.3. It results

$$
\Psi(s)=\frac{(1+|s|)^{2 \gamma}-1}{2 \gamma}-|s|, \quad \forall s \in \mathbf{R} .
$$

Moreover, since $2 \gamma>1$, by Young inequality we get

$$
|s| \leq \frac{1}{4 \gamma}(1+|s|)^{2 \gamma}+c_{1}
$$

where $c_{1}=\frac{2^{1 /(2 \gamma-1)}}{(2 \gamma)^{\prime}}$. Hence from the previous inequality and (3.11), we deduce

$$
\Psi(s) \geq \frac{1}{4 \gamma}(1+|s|)^{2 \gamma}-c_{2}, \quad \forall s \in \mathbf{R},
$$

with $c_{2}=c_{1}+\frac{1}{2 \gamma}$. Let $u_{n}$ be as $(3.1)$ and choose $v_{n}=\left[\left(1+\left|u_{n}\right|\right)^{2 \gamma-1}-1\right] \operatorname{sign}\left(u_{n}\right)$ as a test function in (3.1). Recalling that $u_{n}(x, 0)=u_{0}(x)$, we get

$$
\begin{aligned}
& \int_{\Omega} \Psi\left(u_{n}(x, t)\right) d x+\varrho_{1}(2 \gamma-1) \int_{0}^{t} \int_{\Omega}\left|\nabla u_{n}\right|^{2}\left(1+\left|u_{n}\right|\right)^{(2 \gamma-2)} d x d \tau \\
& \quad \leq \int_{0}^{t} \int_{\Omega}|f|\left|v_{n}\right| d x d \tau+\lambda \int_{0}^{t} \int_{\Omega} \frac{\left(1+\left|u_{n}\right|\right)^{2 \gamma}}{|x|^{2}+\frac{1}{n}} d x d \tau+\int_{\Omega}\left[1+\left|u_{0}(x)\right|\right]^{2 \gamma} d x
\end{aligned}
$$


where $0<t \leq T$. We estimate now the terms in the estimate above. We have

$$
\begin{aligned}
& \lambda \int_{0}^{t} \int_{\Omega} \frac{\left(1+\left|u_{n}\right|\right)^{2 \gamma}}{|x|^{2}+\frac{1}{n}} d x d \tau=\lambda \int_{0}^{t} \int_{\Omega} \frac{\left[\left(1+\left|u_{n}\right|\right)^{\gamma}-1+1\right]^{2}}{|x|^{2}+\frac{1}{n}} d x d \tau \\
& \leq \lambda \int_{0}^{t} \int_{\Omega} \frac{\left[\left(1+\left|u_{n}\right|\right)^{\gamma}-1\right]^{2}}{|x|^{2}} d x d \tau+2 \lambda \int_{0}^{t} \int_{\Omega} \frac{\left[\left(1+\left|u_{n}\right|\right)^{\gamma}-1\right]}{|x|^{2}} d x d \tau \\
& \quad+\lambda \int_{0}^{t} \int_{\Omega} \frac{1}{|x|^{2}} d x d \tau .
\end{aligned}
$$

Notice that it results

$2 \lambda \int_{0}^{t} \int_{\Omega} \frac{\left[\left(1+\left|u_{n}\right|\right)^{\gamma}-1\right]}{|x|^{2}} d x d \tau \leq \varepsilon \int_{0}^{t} \int_{\Omega} \frac{\left[\left(1+\left|u_{n}\right|\right)^{\gamma}-1\right]^{2}}{|x|^{2}} d x d \tau+c_{3} \int_{0}^{t} \int_{\Omega} \frac{1}{|x|^{2}} d x d \tau$,

where $\varepsilon$ will be determined later and $c_{3}=\frac{\lambda^{2}}{\varepsilon}$. Hence from the previous inequalities and by means of Hardy's inequality, we get

$$
\begin{aligned}
& \lambda \int_{0}^{t} \int_{\Omega} \frac{\left(1+\left|u_{n}\right|\right)^{2 \gamma}}{|x|^{2}+\frac{1}{n}} d x d \tau \\
& \leq(\lambda+\varepsilon) \int_{0}^{t} \int_{\Omega} \frac{\left[\left(1+\left|u_{n}\right|\right)^{\gamma}-1\right]^{2}}{|x|^{2}} d x d \tau+\left(\lambda+c_{3}\right) \int_{0}^{t} \int_{\Omega} \frac{1}{|x|^{2}} d x d \tau \\
& \quad \leq \frac{\lambda+\varepsilon}{\Lambda_{N}} \int_{0}^{t} \int_{\Omega}\left|\nabla\left\{\left[\left(1+\left|u_{n}\right|\right)^{\gamma}-1\right] \operatorname{sign}\left(u_{n}\right)\right\}\right|^{2} d x d \tau \\
& \quad+\left(\lambda+c_{3}\right) \int_{0}^{t} \int_{\Omega} \frac{1}{|x|^{2}} d x d \tau .
\end{aligned}
$$

Applying Hölder's inequality, (3.12) and the previous inequalities, we obtain

$$
\begin{aligned}
& \frac{1}{4 \gamma} \sup _{[0, T]} \int_{\Omega}\left(1+\left|u_{n}\right|\right)^{2 \gamma} d x \\
& \quad+\left(\frac{\varrho_{1}(2 \gamma-1)}{\gamma^{2}}-\frac{\lambda+\varepsilon}{\Lambda_{N}}\right) \int_{0}^{T} \int_{\Omega}\left|\nabla\left\{\left[\left(1+\left|u_{n}\right|\right)^{\gamma}-1\right] \operatorname{sign}\left(u_{n}\right)\right\}\right|^{2} d x d \tau \\
& \leq\|f\|_{L^{r}\left(0, T ; L^{q}(\Omega)\right)}\left(\int_{0}^{T}\left[\int_{\Omega}\left(1+\left|u_{n}\right|\right)^{(2 \gamma-1) q^{\prime}} d x\right]^{\frac{r^{\prime}}{q^{\prime}}} d \tau\right)^{\frac{1}{r^{\prime}}} \\
& \quad+c_{4}+\left(\lambda+c_{3}\right) \int_{0}^{T} \int_{\Omega} \frac{1}{|x|^{2}} d x d \tau \\
& \leq c_{5}+c_{f}\left(\int_{0}^{T}\left\|\left(1+\left|u_{n}\right|\right)\right\|_{L^{(2 \gamma-1) q^{\prime}}}^{(2 \gamma-1) r^{\prime}} d \tau\right)^{\frac{1}{r^{\prime}}},
\end{aligned}
$$

where $c_{4}=c_{2}|\Omega|+\int_{\Omega}\left(1+\left|u_{0}\right|\right)^{2 \gamma}, c_{5}=c_{4}+\left(\lambda+c_{3}\right) \int_{0}^{T} \int_{\Omega} \frac{1}{|x|^{2}} d x d \tau$ and $c_{f}=\|f\|_{L^{r}\left(0, T ; L^{q}(\Omega)\right)}$. Denoting by

$$
A=\left(\int_{0}^{T}\left\|\left(1+\left|u_{n}\right|\right)\right\|_{L^{(2 \gamma-1) q^{\prime}}}^{(2 \gamma-1) r^{\prime}} d \tau\right)^{\frac{1}{r^{\prime}}}
$$


and thanks to Sobolev's inequality, we get

$$
\begin{gathered}
\sup _{[0, T]} \int_{\Omega}\left(1+\left|u_{n}\right|\right)^{2 \gamma} d x \leq 4 \gamma\left(c_{5}+c_{f} A\right), \\
\int_{0}^{T}\left[\int_{\Omega}\left[\left(1+\left|u_{n}\right|\right)^{\gamma}-1\right]^{2^{*}} d x\right]^{\frac{2}{2^{*}}} d \tau \leq C_{S}^{-2}\left(\frac{\varrho_{1}(2 \gamma-1)}{\gamma^{2}}-\frac{\lambda+\varepsilon}{\Lambda_{N}}\right)^{-1}\left(c_{5}+c_{f} A\right) .
\end{gathered}
$$

Moreover it is easy to prove the existence of two positive constants $c_{6}$ and $c_{7}$ (for example $c_{6}=c_{7}=2^{2^{*}}$ ) such that

$$
\left[\left(1+\left|u_{n}\right|\right)^{\gamma}-1\right]^{2^{*}} \geq c_{6}\left(1+\left|u_{n}\right|\right)^{2^{*} \gamma}-c_{7}
$$

Hence assuming that

$$
\frac{\varrho_{1}(2 \gamma-1)}{\gamma^{2}}-\frac{\lambda}{\Lambda_{N}}>0,
$$

and choosing $\varepsilon$ such that

$$
\frac{\varrho_{1}(2 \gamma-1)}{\gamma^{2}}-\frac{\lambda+\varepsilon}{\Lambda_{N}}>0
$$

we can conclude that

$$
\int_{0}^{T}\left[\int_{\Omega}\left(1+\left|u_{n}\right|\right)^{2^{*} \gamma} d x\right]^{\frac{2}{2^{*}}} d \tau \leq c_{8}+c_{9} A
$$

where $c_{8}=\left(\frac{1}{c_{6}}\right)^{\frac{2}{2^{*}}} C_{S}^{-2}\left[\frac{\varrho_{1}(2 \gamma-1)}{\gamma^{2}}-\frac{\lambda+\varepsilon}{\Lambda_{N}}\right]^{-1} c_{5}+\left(\frac{c_{7}}{c_{6}}\right)^{\frac{2}{2^{*}}} T$, and $c_{9}=\left(\frac{1}{c_{6}}\right)^{\frac{2}{2^{*}}}$ $C_{S}^{-2}\left[\frac{\varrho_{1}(2 \gamma-1)}{\gamma^{2}}-\frac{\lambda+\varepsilon}{\Lambda_{N}}\right]^{-1} c_{f}$. We distinguish now different cases depending on the size of $q$.

First case: Assume that

$$
1<q<\frac{N r}{N+2(r-1)}, \quad \text { (zone E in Fig. 1). }
$$

Notice that it results

$$
1<q<\frac{N r}{N+2(r-1)} \Rightarrow\left\{\begin{array}{l}
q<r \\
\frac{r^{\prime}}{q^{\prime}}<\frac{2}{2^{*}} .
\end{array}\right.
$$

Hence, we can apply Hölder's inequality to estimate $A$ and we get

$$
A \leq T^{\frac{1}{r^{\prime}}-\frac{2^{*}}{2 q^{\prime}}}\left[\int_{0}^{T}\left(\int_{\Omega}\left(1+\left|u_{n}\right|\right)^{(2 \gamma-1) q^{\prime}} d x\right)^{\frac{2}{2 *}} d \tau\right]^{\frac{2^{*}}{2 q^{\prime}}} .
$$

Thus from (3.17), we deduce that

$\int_{0}^{T}\left[\int_{\Omega}\left[\left(1+\left|u_{n}\right|\right)^{2^{*} \gamma}\right] d x\right]^{\frac{2}{2^{*}}} d \tau \leq c_{8}+c_{10}\left[\int_{0}^{T}\left(\int_{\Omega}\left(1+\left|u_{n}\right|\right)^{(2 \gamma-1) q^{\prime}} d x\right)^{\frac{2}{2^{*}}} d \tau\right]^{\frac{2^{*}}{2 q^{\prime}}}$,

where $c_{10}=c_{9} T^{\frac{1}{r^{\prime}}-\frac{2^{*}}{2 q^{\prime}}}$. Since $q<\frac{N}{2}$ it results $\frac{2^{*}}{2 q^{\prime}}<1$. If we choose $2^{*} \gamma=$ $(2 \gamma-1) q^{\prime}$, that is, $\gamma=\gamma_{1}=\frac{1}{2} \cdot \frac{q(N-2)}{(N-2 q)},(3.15)$ becomes 


$$
\lambda<\lambda_{1} \equiv \frac{\varrho_{1}\left(2 \gamma_{1}-1\right)}{\gamma_{1}^{2}} \Lambda_{N}
$$

and we get

$$
\int_{0}^{T}\left(\int_{\Omega}\left(1+\left|u_{n}\right|\right)^{\left(2 \gamma_{1}-1\right) q^{\prime}} d x\right)^{\frac{2}{2^{*}}} d \tau \leq c_{11}
$$

where $c_{11}$ depends only on $c_{8}, c_{10}, N$ and $q$. Since obviously, it results

$$
\left(1+\left|u_{n}\right|\right)^{2 \gamma} \geq\left|\left[\left(1+\left|u_{n}\right|\right)^{\gamma}-1\right] \operatorname{sign}\left(u_{n}\right)\right|^{2},
$$

using (3.21), (3.20) in (3.13) and by Lemma 2.5 we deduce that (3.10) holds with $\gamma=\gamma_{*}$ and $\lambda<\lambda_{*}$ when (3.18) holds true. Notice that (3.21) is equivalent to

$$
\left\|1+\left|u_{n}\right|\right\|_{L^{p}\left(0, T ; L^{q^{* *}}(\Omega)\right)} \leq c_{12},
$$

with $p=\frac{q(N-2)}{(N-2 q)}>q$ and $c_{12}=c_{11}^{\frac{1}{q^{* *}}}$. Hence it follows

$$
\left\|u_{n}\right\|_{L^{p}\left(0, T ; L^{q^{* *}}(\Omega)\right)} \leq c_{12} .
$$

If otherwise

$$
2^{*} \gamma>(2 \gamma-1) q^{\prime}
$$

that is

$$
\gamma<\gamma_{1}
$$

proceeding as above we get again that (3.10) holds for every $\gamma$ and $\lambda$ satisfying (3.22) and (3.15). Notice that (3.15) is equivalent to require

$$
\frac{\Lambda_{N} \varrho_{1}-\sqrt{\Lambda_{N} \varrho_{1}\left(\Lambda_{N} \varrho_{1}-\lambda\right)}}{\lambda}<\gamma<\frac{\Lambda_{N} \varrho_{1}+\sqrt{\Lambda_{N} \varrho_{1}\left(\Lambda_{N} \varrho_{1}-\lambda\right)}}{\lambda} .
$$

Moreover it results

$$
\frac{\Lambda_{N} \varrho_{1}-\sqrt{\Lambda_{N} \varrho_{1}\left(\Lambda_{N} \varrho_{1}-\lambda\right)}}{\lambda}<\gamma_{1},
$$

if $\lambda \geq \frac{\Lambda_{N} \varrho_{1}}{\gamma_{1}}$ or if $\lambda<\min \left\{\frac{\Lambda_{N} \varrho_{1}}{\gamma_{1}}, \lambda_{1}\right\}$. Observe that the condition $\frac{\Lambda_{N} \varrho_{1}}{\gamma_{1}} \leq \lambda<$ $\Lambda_{N} \varrho_{1}$ can be fullfilled only if $\gamma_{1}>1$, that is if

$$
q>\frac{2 N}{N+2} .
$$

Finally notice that

$$
\gamma_{1} \geq \frac{\Lambda_{N} \varrho_{1}+\sqrt{\Lambda_{N} \varrho_{1}\left(\Lambda_{N} \varrho_{1}-\lambda\right)}}{\lambda} \Leftrightarrow \lambda \geq \lambda_{1} .
$$

Hence we can conclude that if

$$
\frac{2 N}{N+2}<q<\frac{N r}{N+2(r-1)}, \quad \text { (zone } 4 \text { in Fig. 2) }
$$


and $\lambda_{1}=\max \left\{\frac{\Lambda_{N} \varrho_{1}}{\gamma_{1}}, \lambda_{1}\right\}<\lambda<\Lambda_{N} \varrho_{1}$, (3.10) is satisfied for every $\gamma<\gamma^{*} \equiv$ $\frac{\Lambda_{N} \varrho_{1}+\sqrt{\Lambda_{N} \varrho_{1}\left(\Lambda_{N} \varrho_{1}-\lambda\right)}}{\lambda}=\min \left\{\gamma_{1}, \frac{\Lambda_{N} \varrho_{1}+\sqrt{\Lambda_{N} \varrho_{1}\left(\Lambda_{N} \varrho_{1}-\lambda\right)}}{\lambda}\right\}$. We point out that $\gamma^{*}>1$, being $\lambda<\Lambda_{N} \varrho_{1}$.

Second case: Assume now that

$$
\frac{N r}{N+2(r-1)} \leq q<\frac{N}{2} r^{\prime}, \quad \text { (zone C in Fig. 1). }
$$

In this case we will choose $\gamma$ satisfying

$$
2 \gamma \leq(2 \gamma-1) q^{\prime} \leq 2^{*} \gamma,
$$

and we define $\theta \in(0,1)$ as

$$
\frac{1}{(2 \gamma-1) q^{\prime}}=\frac{1-\theta}{2 \gamma}+\frac{\theta}{2^{*} \gamma} .
$$

Then it follows

$$
\begin{aligned}
\left\|\left(1+\left|u_{n}\right|\right)\right\|_{L^{(2 \gamma-1) q^{\prime}}}^{r^{\prime}(2 \gamma-1)} & \leq\left\|\left(1+\left|u_{n}\right|\right)\right\|_{L^{2 \gamma}}^{(1-\theta) r^{\prime}(2 \gamma-1)}\left\|\left(1+\left|u_{n}\right|\right)\right\|_{L^{2^{*} \gamma}}^{\theta r^{\prime}(2 \gamma-1)} \\
& \leq\left\|\left(1+\left|u_{n}\right|\right)\right\|_{L^{\infty}\left(0, T ; L^{2 \gamma}(\Omega)\right)}^{(1-\theta) r^{\prime}(2 \gamma-1)}\left(\int_{\Omega}\left(1+\left|u_{n}\right|\right)^{2^{*} \gamma} d x\right)^{\frac{\theta r^{\prime}(2 \gamma-1)}{2^{*} \gamma}} .
\end{aligned}
$$

Integrating on time we obtain

$$
\begin{aligned}
& \int_{0}^{T}\left\|\left(1+\left|u_{n}\right|\right)\right\|_{L^{(2 \gamma-1) q^{\prime}}}^{r^{\prime}(2 \gamma-1)} d \tau \\
& \quad \leq\left\|\left(1+\left|u_{n}\right|\right)\right\|_{L^{\infty}\left(0, T ; L^{2 \gamma}(\Omega)\right)}^{2 \gamma \mu_{1}} \int_{0}^{T}\left(\int_{\Omega}\left(1+\left|u_{n}\right|\right)^{2^{*} \gamma} d x\right)^{\frac{2}{2^{*} \mu_{2}}} d \tau,
\end{aligned}
$$

with

$$
\mu_{1}=\frac{(1-\theta) r^{\prime}(2 \gamma-1)}{2 \gamma} \text { and } \quad \mu_{2}=\frac{\theta r^{\prime}(2 \gamma-1)}{2 \gamma}
$$

We need

$$
\mu_{2} \leq 1 \quad \Leftrightarrow \quad \theta \leq \frac{2 \gamma}{r^{\prime}(2 \gamma-1)} .
$$

By (3.29) we know that

$$
\theta=\left[\frac{1}{2 \gamma}-\frac{1}{(2 \gamma-1) q^{\prime}}\right] N \gamma
$$

hence the condition (3.32) becomes

$$
2 \gamma\left[N q^{\prime} r^{\prime}-N r^{\prime}-2 q^{\prime}\right] \leq q^{\prime} r^{\prime} N
$$

Since $N \geq 3$, it results

$$
N q^{\prime} r^{\prime}-N r^{\prime}-2 q^{\prime} \leq 0 \quad \Leftrightarrow \quad q \geq \frac{N}{2} r^{\prime} .
$$

Recall that we are assuming $q<\frac{N}{2} r^{\prime}$; thus (3.34) is satisfied if (and only if )

$$
\gamma \leq \frac{1}{2} \cdot \frac{q r N}{N r-2 q(r-1)}=\gamma_{2}
$$


With such a choice we get

$$
\begin{aligned}
& \int_{0}^{T}\left\|\left(1+\left|u_{n}\right|\right)\right\|_{L^{(2 \gamma-1) q^{\prime}}}^{r^{\prime}(2 \gamma-1)} d \tau \\
& \quad \leq c_{13}\left(\sup _{[0, T]} \int_{\Omega}\left(1+\left|u_{n}\right|\right)^{2 \gamma} d x\right)^{\mu_{1}}\left(\int_{0}^{T}\left[\int_{\Omega}\left(1+\left|u_{n}\right|\right)^{2^{*} \gamma} d x\right]^{\frac{2}{2^{*}}} d \tau\right)^{\mu_{2}},
\end{aligned}
$$

with $\mu_{2} \leq 1$ and $c_{13}=T^{1-\mu_{2}}$. By estimates (3.14) and (3.17) and the previous one, we obtain

$$
A^{r^{\prime}} \leq c_{13}\left[4 \gamma\left(c_{5}+c_{f} A\right)\right]^{\mu_{1}}\left(c_{8}+c_{9} A\right)^{\mu_{2}} \leq c_{14}+c_{15} A^{\beta},
$$

with $\beta=\mu_{1}+\mu_{2}=r^{\prime} \frac{(2 \gamma-1)}{2 \gamma}<r^{\prime}$ and $c_{14}$ and $c_{15}$ positive constants independent on $n$. Therefore it follows

$$
\int_{0}^{T}\left(\int_{\Omega}\left(1+\left|u_{n}\right|\right)^{(2 \gamma-1) q^{\prime}} d x\right)^{\frac{r^{\prime}}{q^{\prime}}} d \tau \leq c_{16},
$$

where $c_{16}$ is independent on $n$ and with $\gamma$ satisfying (3.15) and (3.36). Observe that the previous inequality implies

$$
\int_{0}^{T}\left(\int_{\Omega}\left|u_{n}\right|^{(2 \gamma-1) q^{\prime}} d x\right)^{\frac{r^{\prime}}{q^{\prime}}} d \tau \leq c_{16}
$$

Using (3.37) and proceeding as in the previous case, it follows that (3.10) holds true for every $\gamma$ satisfying (3.15) and (3.36). Notice that if we choose $\gamma=\gamma_{2}$ (that is, the larger value that we can choose for $\gamma$ when the summability exponents of $f$ are in the zone $C$ ), condition (3.36) is obviously satisfied and (3.15) becomes $\lambda<\lambda_{2}$. Thus (3.10) is proved with $\gamma=\gamma_{*}\left(\gamma_{*}\right.$ as in (1.25)) when $\lambda<\lambda_{*}$ and (3.27) holds true.

As noticed, condition (3.15) is equivalent to (3.23). Moreover, it results

$$
\frac{\Lambda_{N} \varrho_{1}-\sqrt{\Lambda_{N} \varrho_{1}\left(\Lambda_{N} \varrho_{1}-\lambda\right)}}{\lambda}<\gamma_{2},
$$

if $\lambda \geq \frac{\Lambda_{N} \varrho_{1}}{\gamma_{2}}$ or if $\lambda<\min \left\{\frac{\Lambda_{N} \varrho_{1}}{\gamma_{2}}, \lambda_{2}\right\}$. Observe that the condition $\frac{\Lambda_{N} \varrho_{1}}{\gamma_{2}} \leq \lambda<$ $\Lambda_{N} \varrho_{1}$ can be fulfilled only if $\gamma_{2}>1$, that is if

$$
q>\frac{2 N r}{N r+4(r-1)}, \quad \text { (zones } 2 \text { and } 3 \text { in Fig. 2) }
$$

Finally, notice that

$$
\gamma_{2} \geq \frac{\Lambda_{N} \varrho_{1}+\sqrt{\Lambda_{N} \varrho_{1}\left(\Lambda_{N} \varrho_{1}-\lambda\right)}}{\lambda} \Leftrightarrow \lambda \geq \lambda_{2} .
$$

Hence we can conclude that if (3.27) and (3.38) hold and $\lambda_{2}=\max \left\{\frac{\Lambda_{N} \varrho_{1}}{\gamma_{2}}, \lambda_{2}\right\}<$ $\lambda<\Lambda_{N} \varrho_{1}$, then (3.10) is satisfied for every $\gamma<\gamma^{*} \equiv \frac{\Lambda_{N} \varrho_{1}+\sqrt{\Lambda_{N} \varrho_{1}\left(\Lambda_{N} \varrho_{1}-\lambda\right)}}{\lambda}=$ 
$\min \left\{\gamma_{2}, \frac{\Lambda_{N} \varrho_{1}+\sqrt{\Lambda_{N} \varrho_{1}\left(\Lambda_{N} \varrho_{1}-\lambda\right)}}{\lambda}\right\}$ where, as noticed before, $\gamma^{*}>1$ being $\lambda<$ $\Lambda_{N} \varrho_{1}$.

Third case: Assume that (1.20) holds true, that is,

$$
q \geq \frac{N}{2} r^{\prime}, \quad \text { (zone A in Fig. 1). }
$$

In this case, by (3.35) it follows that (3.34) is satisfied for every value of $\gamma>\frac{1}{2}$ and proceeding as in the previous case, we deduce that (3.10) is satisfied for every $\gamma$ and $\lambda$ satisfying (3.15), that is for every $0<\lambda<\Lambda_{N} \varrho_{1}$ and for every $\gamma \in\left(\frac{1}{2}, \frac{\Lambda_{N} \varrho_{1}+\sqrt{\Lambda_{N} \varrho_{1}\left(\Lambda_{N} \varrho_{1}-\lambda\right)}}{\lambda}\right)$. Notice that the subset $\left(\frac{1}{2}, \frac{\Lambda_{N} \varrho_{1}+\sqrt{\Lambda_{N} \varrho_{1}\left(\Lambda_{N} \varrho_{1}-\lambda\right)}}{\lambda}\right)$ is nonempty, being $\frac{\Lambda_{N} \varrho_{1}+\sqrt{\Lambda_{N} \varrho_{1}\left(\Lambda_{N} \varrho_{1}-\lambda\right)}}{\lambda}>1$.

Hence we have proved that (3.10) holds for every $\gamma$ as in the statement of Theorem 1.3.

Second step. As said before, the assertion will follow once proved that the sequence $u_{n}$ tends to a solution $\mathrm{u}$ of (1.1) and that it is possible to pass to the limit on $\mathrm{n}$ in (3.10). To this aim, we notice that if (1.20) or (1.26) holds true and $0<\lambda<\Lambda_{N} \varrho_{1}$, being in these cases $\gamma>1$, by (3.10) we deduce that $\nabla u_{n}$ is equibounded in $\left(L^{2}(Q)\right)^{N}$. Otherwise, i.e. if both the inequalities (1.20) and (1.26) are not satisfied and $0<\lambda<\lambda_{*}$, by Young inequality, we deduce that

$$
\begin{aligned}
\int_{Q}\left|\nabla u_{n}\right|^{M} d x d t \leq & \int_{Q}\left|\nabla u_{n}\right|^{2}\left(1+\left|u_{n}\right|\right)^{2\left(\gamma_{*}-1\right)} d x d t \\
& +\int_{Q}\left(1+\left|u_{n}\right|\right)^{\frac{2\left(1-\gamma_{*}\right)}{M(2-M)}} d x d t
\end{aligned}
$$

from which follows that there exists a constant $C$ independent of $\mathrm{n}$ such that

$$
\int_{Q}\left|\nabla u_{n}\right|^{M} d x d t \leq C
$$

if we choose $\frac{2\left(1-\gamma_{*}\right)}{M(2-M)}=2 \gamma_{*} \frac{N+2}{N}$, that is, $M=1+\sqrt{1-\frac{N\left(1-\gamma_{*}\right)}{\gamma_{*}(N+2)}}$ (since by (3.10) with such a choice, the right hand side of (3.41) is equibounded). Notice that it results $M>1$. Defining

$$
\bar{M}= \begin{cases}2, & \text { if }(1.26) \text { or }(1.20) \text { holds true and } 0<\lambda<\Lambda_{N} \varrho_{1} \\ M, & \text { otherwise, i.e. if both the inequalities } \\ & (1.20) \text { and }(1.26) \text { are not satisfied and } 0<\lambda<\lambda_{*}\end{cases}
$$

the previous estimates on $\nabla u_{n}$ become

$$
\int_{Q}\left|\nabla u_{n}\right|^{\bar{M}} d x d t \leq C .
$$

Hence, by the assumption (1.3) it follows that also $a(x, t, u, \nabla u)$ is equibounded in $L^{\bar{M}}(Q)$. 
Finally notice that estimate (3.10) implies also the following

$$
\lambda \frac{u_{n}}{|x|^{2}} \quad \text { is equibounded in } \quad L^{h}(Q), \quad \forall 1 \leq h<\frac{2 \gamma N}{2(2 \gamma-1)+N} .
$$

As a matter of fact it results

$$
\iint_{Q}\left(\frac{\lambda\left|u_{n}\right|}{|x|^{2}}\right)^{h} \leq \lambda^{h}\left[\iint_{Q} \frac{\left|u_{n}\right|^{2 \gamma}}{|x|^{2}}+\iint_{Q} \frac{1}{|x|^{\delta}}\right]
$$

where $\delta=\frac{2 h(2 \gamma-1)}{2 \gamma-h}$ and the two integrals in the right hand side are finite by (3.10) if $\mathrm{h}$ is as in (3.45).

Hence applying Lemma 2.8 (with $X=W_{0}^{1, \bar{M}}(\Omega), \mathrm{B}=L^{\bar{M}}(\Omega)$ and $Y=$ $W^{-1, \alpha}(\Omega)$, where $\left.\alpha=\min \left\{h^{*}, \bar{M}\right\}\right)$ we deduce that there exists a subsequence of $u_{n}$, that we denote again $u_{n}$, such that the following limits hold true

$$
\begin{gathered}
u_{n} \rightarrow u, \quad \text { strongly in } L^{\bar{M}}(Q), \\
u_{n} \rightarrow u, \quad \text { a.e. in } Q, \\
a\left(x, t, u_{n}, \nabla u_{n}\right) \rightarrow \chi, \quad \text { weakly in } L^{\bar{M}}(Q), \\
\lambda \frac{\left|u_{n}\right|}{|x|^{2}} \rightarrow \lambda \frac{|u|}{|x|^{2}}, \quad \text { weakly in } L^{h}(Q) .
\end{gathered}
$$

To conclude the proof we need to show the a.e. convergence of $\nabla u_{n}$ to $\nabla u$ in $Q$. This allows (together with the previous convergences) to pass to the limit in (3.1) and to conclude that $\mathrm{u}$ is a solution of (1.1) which satisfies the regularity properties in Theorem 1.3. To this aim, we observe that if $\bar{M}=2$ it is sufficient to apply Theorem 4.1 in [12]. If otherwise $\bar{M}=M<2$, taking $T_{k}\left(u_{n}\right)$ as a test function in (3.1) (where $T_{k}$ is the truncation function defined in (3.2)) and using (3.45) we deduce that

$$
T_{k}\left(u_{n}\right) \text { is equibounded in } L^{2}\left(0, T ; H_{0}^{1}(\Omega)\right) \text {. }
$$

Hence the convergence a.e. of the gradients follows proceeding as in [9].

Remark 3.1. Notice that $\lambda_{1}=\lambda_{2}$ if and only if $q=\frac{N r}{N+2(r-1)}$, that is, there is continuity in the results.

Observe also that

$$
C(\gamma, \lambda)=\left(\frac{(2 \gamma-1)}{\gamma^{2}}-\frac{\varrho_{1} \lambda}{\Lambda_{N}}\right)
$$

is an increasing function in $\gamma$ if $\gamma>1$ and a decreasing function in $\gamma$ if $\gamma<1$. We have $\gamma_{1}(q) \geq 1$ if and only if $q \geq \frac{2 N}{N+2}$ and $\gamma_{2}(q) \geq 1$ if and only if $\frac{N}{2 q}+\frac{1}{r} \leq 1+\frac{N}{4}$. Since $1 \leq \gamma_{2}(q) \leq \gamma_{1}(q)$ in zone 2 and 3 of Fig. $2, \lambda_{1}(1)=0$ and $\lambda_{1}\left(\frac{2 N}{N+2}\right)=\varrho_{1}\left(\frac{N-2}{2}\right)^{2}$, it follows that in zone 2,3, and 4 of Fig. $2, u \in V^{2}(Q)$, for all $0<\lambda<\varrho_{1} \Lambda_{N}$.

Remark 3.2. By the estimate (3.44) it follows that $\nabla u \in\left(L^{2}(Q)\right)^{N}$ if $(1.26)$ or (1.20) holds true and $0<\lambda<\Lambda_{N} \varrho_{1}$; otherwise (i.e. in all the other cases considered here) $\nabla u \in\left(L^{M}(Q)\right)^{N}$, with $M$ as in (3.42), i.e. $M=1+\sqrt{1-\frac{N\left(1-\gamma_{*}\right)}{\gamma_{*}(N+2)}}$. 
Remark 3.3. We notice that all the results of Theorems 1.2 and 1.3 are true if we replace in (1.1) the Hardy potential $\lambda \frac{u}{|x|^{2}}$ with the nonlinear term $g(x, t, u, \nabla u)$ where $g(x, t, \sigma, \xi): Q \times \mathbf{R} \times \mathbf{R}^{N} \rightarrow \mathbf{R}$ is a Caratheodory function satisfying

$$
|g(x, t, s, \xi)| \leq \lambda \frac{|s|}{|x|^{2}} .
$$

As a matter of fact, in this case it is sufficient to replace the approximating problems (3.1) with

$$
\left\{\begin{array}{l}
\left(u_{n}\right)_{t}-\operatorname{div}\left(a\left(x, t, u_{n}, \nabla u_{n}\right)\right)=g_{n}\left(x, t, u_{n}, \nabla u_{n}\right)+T_{n}(f) \quad \text { in } Q, \\
u_{n}(x, t)=0 \quad \text { on } \partial \Omega \times(0, T), \\
u_{n}(x, 0)=u_{0}(x) \quad \text { in } \Omega,
\end{array}\right.
$$

where

$$
g_{n}(x, t, \sigma, \xi)=\frac{g(x, t, \sigma, \xi)}{1+\frac{1}{n}|g(x, t, \sigma, \xi)|},
$$

and since it results

$$
\left|g_{n}(x, t, \sigma, \xi)\right| \leq n
$$

and

$$
\left|g_{n}(x, t, \sigma, \xi)\right| \leq|g(x, t, \sigma, \xi)| \leq \lambda \frac{|s|}{|x|^{2}},
$$

both the proofs of Theorems 1.2 and 1.3 can easily adapted to this more general case.

\section{A different point of view for further regularity results}

In this section we give further regularity results. For simplicity, we consider the model problem (1.5), i.e.

$$
\begin{cases}u_{t}-\Delta u=\lambda \frac{u}{|x|^{2}}+f \quad \text { in } Q, \\ u(x, t)=0 \quad \text { on } \partial \Omega \times(0, T), \\ u(x, 0)=0 \quad \text { in } \Omega .\end{cases}
$$

As just noticed, differently from the case $\lambda=0$, the presence of the singular potential in (4.1) produces unbounded solutions for every choice of regular (nonzero) and nonnegative datum $f$.

Anyway, the regularity of $f$ influences the regularity of $u$ and in particular, it may cause the boundedness of $|x|^{\alpha_{1}} u$ (for sufficiently summable $f$ ) where $\alpha_{1}$ is as before (see (1.8)). As a matter of fact we have the following result.

Theorem 4.1. Assume $0<\lambda \leq \Lambda_{N}$ and $f \in L^{r}\left(0, T ; L^{q}(\Omega)\right)$ with $r$, $q$ satisfying

$$
\frac{1}{r}+\frac{N}{2 q}=1-\chi_{1}, \quad \chi_{1} \in(0,1), \quad(\text { zone } A \text { in Fig. } 1) .
$$

If $u \in V_{2}(Q)$ is a solution to (4.1), then $|x|^{\alpha_{1}} u \in L^{\infty}(Q)$. 
Remark 4.2. The proof of Theorem 4.1 is not linear in nature. As a matter of fact, it works also replacing the linear problem (4.1) with the following nonlinear problem

$$
\begin{cases}u_{t}-\Delta u=g(x, t, u, \nabla u)+f & \text { in } Q, \\ u(x, t)=0 & \text { on } \partial \Omega \times(0, T), \\ u(x, 0)=0 & \text { in } \Omega,\end{cases}
$$

where $g(x, t, \sigma, \xi): Q \times \mathbf{R} \times \mathbf{R}^{N} \rightarrow \mathbf{R}$ is as in Remark 3.3, i.e. a Caratheodory function satisfying

$$
|g(x, t, s, \xi)| \leq \lambda \frac{|s|}{|x|^{2}} .
$$

Moreover the result of the Theorem 4.1, still holds for nonzero initial data $u_{0}(x)$ satisfying $|x|^{\alpha_{1}} u_{0} \in L^{\infty}(\Omega)$.

Moreover, if $f$ is less summable, we have the following result.

Theorem 4.3. Assume $0<\lambda \leq \Lambda_{N}$ and $|x|^{-h} f \in L^{r}\left(0, T ; L^{q}(\Omega)\right)$, where

$$
\frac{1}{r}+\frac{N}{2 q}>1, \quad r \in(1,+\infty], \quad q \in(1,+\infty),
$$

and

$$
h= \begin{cases}h_{2}=\frac{\alpha_{1}}{\gamma_{2}^{\prime}} & \text { if } \frac{N r}{N+2(r-1)} \leq q<\frac{N}{2} r^{\prime},(\text { zone C in Fig. } 1), \\ h_{1}=\alpha_{1} \frac{2 N-q(N+2)}{q(N-2)} & \text { if } \left.1<q<\frac{N r}{N+2(r-1)}, \quad \text { (zone E in Fig. } 1\right) .\end{cases}
$$

Then there exists a weak solution $u$ of (4.1) satisfying

$$
\left[\left(1+|x|^{\alpha_{1}}|u|\right)^{\gamma}-1\right] \operatorname{sign}(u) \in V_{\alpha_{1}}^{2}(Q) \cap L^{\delta}\left(0, T ; L_{\alpha_{1}}^{m}(\Omega)\right),
$$

where $\gamma>\frac{1}{2}$ is as in (1.25), i.e.

$$
\gamma=\gamma_{*}=\left\{\begin{array}{l}
\gamma_{2}=\frac{1}{2} \frac{q r N}{N r-2 q(r-1)} \quad \text { if } \frac{N r}{N+2(r-1)} \leq q<\frac{N}{2} r^{\prime},(\text { zone C in Fig. 1), } \\
\gamma_{1}=\frac{1}{2} \frac{q(N-2)}{N-2 q}=\frac{q^{* *}}{2^{*}} \text { if } 1<q<\frac{N r}{N+2(r-1)}, \quad \text { (zone E in Fig. 1), }
\end{array}\right.
$$

$m \in\left[2,2^{*}\right]$ and $\delta=\frac{4 m}{N(m-2)}$. Moreover it results $\gamma_{2} \geq 1$ if and only if

$$
\frac{1}{r}+\frac{N}{2 q} \leq 1+\frac{N}{4}, \quad \text { (zones 2, } 3 \text { in Fig. 2), }
$$

and $\gamma_{1} \geq 1$ if and only if $q \geq \frac{2 N}{N+2}$ (zone 4 in Fig. 2 ) and hence in this case it follows that $u \in V^{2}(Q)$.

In particular, choosing $m=2^{*}$, we get the best summability exponent in the spacial variable (i.e. $2^{*} \gamma$ ) together with the lower summability exponent in the time variable, (i.e. $2 \gamma$ ) and we obtain

$$
|x|^{-h} u \in L^{2 \gamma}\left(0, T ; L^{2^{*} \gamma}(\Omega)\right),
$$


that is

$$
\begin{aligned}
& 2 \gamma=2 \gamma_{1}=p_{1}=\frac{2}{2^{*}} q^{* *}=\frac{q(N-2)}{(N-2 q)}>q, \quad 2^{*} \gamma=2^{*} \gamma_{1}=s_{1} \equiv q^{* *}, \\
& h=h_{1}=\alpha_{1} \frac{2 N-q(N+2)}{q(N-2)}, \\
& \left.\quad \text { if } 1<q<\frac{N r}{N+2(r-1)}, \quad \text { (zona E in the Fig. } 1\right) ;
\end{aligned}
$$

and

$$
\begin{aligned}
2 \gamma=2 \gamma_{2} & =\frac{q r N}{N r-2 q(r-1)}, \quad 2^{*} \gamma=2^{*} \gamma_{2}=\frac{q r N^{2}}{[N r-2 q(r-1)]) N-2)}, \\
h=h_{2} & =\frac{-\alpha_{1}}{\gamma_{2}^{\prime}} \\
& \text { if } \frac{N r}{N+2(r-1)} \leq q<\frac{N}{2} r^{\prime}, \quad \text { (zone C in Fig. 1). }
\end{aligned}
$$

Choosing $m=2 \frac{N+2}{N}$, we get

$$
u \in L^{\bar{s}}(Q), \quad \bar{s}=2 \gamma \frac{N+2}{N},
$$

that is

$$
\bar{s}=\overline{s_{1}} \equiv \frac{q\left(N^{2}-4\right)}{N(N-2 q)},
$$

if (4.9) holds. Otherwise (i.e. if (4.10) holds), then

$$
\bar{s}=\overline{s_{2}} \equiv \frac{q r(N+2)}{N r+2 q-2 q r} .
$$

Moreover in this last case (zone $C$ in Fig. 1), choosing $m=2+4 \frac{q^{\prime}}{r^{\prime} N}$, we get

$$
|x|^{-h} u \in L^{p_{2}}\left(0, T ; L^{s_{2}}(\Omega)\right), \quad p_{2}=\rho r^{\prime}, \quad s_{2}=\rho q^{\prime}, \quad \rho=\frac{r N(q-1)+2 q(r-1)}{r(N-2 q)+2 q} .
$$

Remark 4.4. We recall that for a nonnegative function $f$, the assumption

$$
|x|^{-\alpha_{1}} f \in L^{1}(Q),
$$

is not only a sufficient condition to guarantee the existence of a solution of (4.1) but also a necessary condition as proved in [7]. Moreover, we notice that when $f$ is in the zone $\mathrm{A}$ in Fig. 1 , then $q \geq \frac{2 N}{N+2}$ and hence $f$ satisfies this necessary and sufficient condition (4.13).

Remark 4.5. We notice that also the proof of Theorem 4.3 is not linear in nature. As a matter of fact, it works also replacing the linear problem (4.1) with the nonlinear problem (4.3) with g a Caratheodory function satisfying (4.4). For further details see Remark 5.3. 
Remark 4.6. We notice that if $\lambda \rightarrow 0^{+}$then $\alpha_{1} \rightarrow 0$ and the regularity property (4.6) becomes the same regularity proved in absence of the Hardy potential (see $[14])$.

The proof of the previous results can be found in the next section.

\section{Proof of the further results}

\subsection{Proof of Theorem 4.1}

Consider the following notations,

$$
\begin{aligned}
& A_{k}=\left\{(x, t) \in \Omega \times(0, T):|x|^{\alpha_{1}}|u(x, t)|>k\right\}, \\
& A_{k}^{t}=\left\{x \in \Omega:|x|^{\alpha_{1}}|u(x, t)|>k\right\} .
\end{aligned}
$$

Taking $|x|^{-\alpha_{1}}\left(|x|^{\alpha_{1}}|u|-k\right)_{+} \operatorname{sign}(u)$ as a test function in (4.1) and integrating in the cylinder $(0, t) \times \Omega, t \leq t_{1}$, where $t_{1}$ will be chosen later, we get

$$
\begin{aligned}
\int_{\Omega} & \varphi(u(x, t)) d x+\int_{0}^{t} \int_{\Omega} \nabla u \nabla\left[|x|^{-\alpha_{1}}\left(|x|^{\alpha_{1}}|u|-k\right)_{+} \operatorname{sign}(u)\right] d x d z \\
\leq & \lambda \int_{0}^{t} \int_{A_{k}^{z}} \frac{u(x, z)\left(|x|^{\alpha_{1}}|u(x, z)|-k\right)_{+} \operatorname{sign}(u)}{|x|^{2+\alpha_{1}}} d x d z \\
& +\int_{0}^{t} \int_{A_{k}^{z}}|x|^{-\alpha_{1}}|f|\left(|x|^{\alpha_{1}}|u|-k\right)_{+} d x d z, \text { a.e. } t \in\left(0, t_{1}\right),
\end{aligned}
$$

where $\varphi(s)=\int_{0}^{s}|x|^{-2 \alpha_{1}}\left(|x|^{\alpha_{1}}|\sigma|-k\right)_{+} \operatorname{sign}(\sigma) d \sigma$. Notice that it results,

$$
\begin{aligned}
\int_{0}^{t} & \int_{\Omega} \nabla u \nabla\left[|x|^{-\alpha_{1}}\left(|x|^{\alpha_{1}}|u|-k\right)_{+} \operatorname{sign}(u)\right] d x d z \\
= & \int_{0}^{t} \int_{\Omega}\left[\nabla u \nabla\left(|x|^{-\alpha_{1}}\right)\right]\left(|x|^{\alpha_{1}}|u|-k\right)_{+} \operatorname{sign}(u) d x d z \\
& +\int_{0}^{t} \int_{\Omega} \nabla u|x|^{-\alpha_{1}} \nabla\left[\left(|x|^{\alpha_{1}}|u|-k\right)_{+} \operatorname{sign}(u)\right] d x d z .
\end{aligned}
$$

Since $\lambda=\alpha_{1}\left((N-2)-\alpha_{1}\right)$, we have

$$
\begin{aligned}
0= & \int_{0}^{t} \int_{\Omega} \operatorname{div}\left[\left(\nabla|x|^{-\alpha_{1}}\right) u\left(|x|^{\alpha_{1}}|u|-k\right)_{+} \operatorname{sign}(u)\right] d x d z \\
= & \int_{0}^{t} \int_{\Omega}\left(\nabla|x|^{-\alpha_{1}}\right) u \nabla\left[\left(|x|^{\alpha_{1}}|u|-k\right)_{+} \operatorname{sign}(u)\right] d x d z \\
& +\int_{0}^{t} \int_{\Omega} \operatorname{div}\left[\left(\nabla|x|^{-\alpha_{1}}\right) u\right]\left(|x|^{\alpha_{1}}|u|-k\right)_{+} \operatorname{sign}(u) d x d z \\
= & \int_{0}^{t} \int_{\Omega}\left(\nabla|x|^{-\alpha_{1}}\right) u \nabla\left[\left(|x|^{\alpha_{1}}|u|-k\right)_{+} \operatorname{sign}(u)\right] d x d z
\end{aligned}
$$




$$
\begin{aligned}
& -\lambda \int_{0}^{t} \int_{\Omega} \frac{u}{|x|^{\alpha_{1}+2}}\left(|x|^{\alpha_{1}}|u|-k\right)_{+} \operatorname{sign}(u) d x d z \\
& +\int_{0}^{t} \int_{\Omega}\left[\nabla u \nabla|x|^{-\alpha_{1}}\right]\left(|x|^{\alpha_{1}}|u|-k\right)_{+} \operatorname{sign}(u) d x d z,
\end{aligned}
$$

then we deduce

$$
\begin{aligned}
\int_{0}^{t} & \int_{\Omega}\left[\nabla u \nabla\left(|x|^{-\alpha_{1}}\right)\right]\left(|x|^{\alpha_{1}}|u|-k\right)_{+} \operatorname{sign}(u) d x d z \\
& +\int_{0}^{t} \int_{\Omega}\left(\nabla|x|^{-\alpha_{1}}\right) u \nabla\left[\left(|x|^{\alpha_{1}}|u|-k\right)_{+} \operatorname{sign}(u)\right] d x d z \\
= & \lambda \int_{0}^{t} \int_{\Omega} \frac{u}{|x|^{\alpha_{1}+2}}\left(|x|^{\alpha_{1}}|u|-k\right)_{+} \operatorname{sign}(u) d x d z
\end{aligned}
$$

Since $|x|^{-2 \alpha_{1}} \nabla\left(|x|^{\alpha_{1}} u\right)=|x|^{-\alpha_{1}} \nabla u-u \nabla|x|^{-\alpha_{1}}$, using (5.3) and (5.4) in (5.2), it follows that

$$
\begin{aligned}
& \sup _{t \in\left(0, t_{1}\right)} \int_{\Omega} \varphi(u(x, t)) d x+\int_{0}^{t_{1}} \int_{\Omega}|x|^{-2 \alpha_{1}}\left|\nabla\left(|x|^{\alpha_{1}}|u|-k\right)+\operatorname{sign}(u)\right|^{2} d x d z \\
& \quad \leq \int_{0}^{t_{1}} \int_{A_{k}^{z}}|x|^{-\alpha_{1}}|f|\left(|x|^{\alpha_{1}}|u|-k\right)_{+} d x d z .
\end{aligned}
$$

Moreover, there exists a positive constant $C$ such that

$$
\int_{A_{k}^{t}} \varphi(u(x, t)) d x \geq C \int_{A_{k}^{t}}|x|^{-2 \alpha_{1}}\left(|x|^{\alpha_{1}}|u(x, t)|-k\right)_{+}^{2} d x,
$$

and therefore it follows

$$
\begin{aligned}
& C\left(\left\|\left(|x|^{\alpha_{1}}|u|-k\right)+\right\|_{L^{\infty}\left(0, t_{1} ; L_{\alpha_{1}}^{2}(\Omega)\right)}^{2}+\left\|\left(|x|^{\alpha_{1}}|u|-k\right)+\operatorname{sign}(u)\right\|_{L^{2}\left(0, t_{1} ; W_{0, \alpha_{1}}^{1,2}(\Omega)\right)}^{2}\right. \\
& \leq \int_{0}^{t_{1}} \int_{A_{k}^{z}}|x|^{-\alpha_{1}}|f|\left(|x|^{\alpha_{1}}|u|-k\right)_{+} d x d z .
\end{aligned}
$$

Notice that the term of the right hand in (5.6) can be estimated as follows,

$$
\begin{aligned}
& \int_{0}^{t_{1}} \int_{A_{k}^{z}}|x|^{-\alpha_{1}}|f|\left(|x|^{\alpha_{1}}|u|-k\right)_{+} d x d z \\
& \quad \leq \int_{0}^{t_{1}} \int_{A_{k}^{z}}|x|^{-2 \alpha_{1}}|f|\left(|x|^{\alpha_{1}}|u|-k\right)_{+}^{2} d x d z+\int_{0}^{t_{1}} \int_{A_{k}^{z}}|f| d x d z .
\end{aligned}
$$

Let us study each member of the previous inequality.

On the one hand, applying Hölder's inequality, we have

$$
\begin{aligned}
& \int_{0}^{t_{1}} \int_{A_{k}^{z}}|x|^{-2 \alpha_{1}}|f|\left(|x|^{\alpha_{1}}|u|-k\right)_{+}^{2} d x d z \\
& \quad \leq\|f\|_{L^{r}\left(0, t_{1} ; L^{q}(\Omega)\right)}\left(\int_{0}^{t_{1}}\left[\int_{A_{k}^{z}}|x|^{-2 \alpha_{1} q^{\prime}}\left(|x|^{\alpha_{1}}|u|-k\right)_{+}^{2 q^{\prime}} d x\right]^{\frac{r^{\prime}}{q^{\prime}}} d z\right)^{\frac{1}{r^{\prime}}} \\
& \quad \leq C_{f}\left(\int_{0}^{t_{1}}\left\|\left(|x|^{\alpha_{1}}|u|-k\right)_{+}\right\|_{L_{\alpha_{1}}^{2 q^{\prime}}}^{2 r^{\prime}} d z\right)^{\frac{1}{r^{\prime}}}
\end{aligned}
$$


where $C_{f}=\|f\|_{L^{r}\left(0, T ; L^{q}(\Omega)\right)}$. Let us define,

$$
\bar{r}=2 r^{\prime}, \bar{q}=2 q^{\prime}, \hat{r}=\bar{r}(1+\chi), \hat{q}=\bar{q}(1+\chi), \chi=\frac{2 \chi_{1}}{N} .
$$

Notice that assumption (4.2) is equivalent to require $\frac{1}{\hat{r}}+\frac{N}{2 \hat{q}}=\frac{N}{4}$. Hence it results $\left(N-\alpha_{1} \hat{q}\right)>0$, which implies that $|x|^{-\alpha_{1} \hat{q}} \in L^{1}(\Omega)$. Denote $\mu(k)=$ $\int_{0}^{t_{1}}\left(\int_{A_{k}^{t}}|x|^{-\alpha_{1} \hat{q}} d x\right)^{\frac{\hat{r}}{\hat{q}}} d t$. Applying Hölder's inequality, we obtain

$$
\begin{aligned}
& \int_{0}^{t_{1}} \int_{A_{k}^{z}}|x|^{-2 \alpha_{1}}|f|\left(|x|^{\alpha_{1}}|u|-k\right)_{+}^{2} d x d z \\
& \leq C_{f}\left(\int_{0}^{t_{1}}\left\|\left(|x|^{\alpha_{1}}|u|-k\right)_{+}\right\|_{L_{\alpha_{1}}^{\bar{q}}\left(A_{k}^{z}\right)}^{\overline{\bar{r}}} d z\right)^{\frac{2}{\bar{r}}} \\
& =C_{f}\left[\int_{0}^{t_{1}}\left(\int_{A_{\bar{k}}^{z}}|x|^{-\alpha_{1} \frac{\hat{q}}{1+\chi}}\left|\left(|x|^{\alpha_{1}}|u|-k\right)_{+}\right|^{\frac{\hat{q}}{1+\chi}} d x\right)^{\frac{\hat{r}}{\hat{q}}} d z\right]^{\frac{2(1+\chi)}{\hat{r}}} \\
& \leq C_{f}\left[\int_{0}^{t_{1}}\left(\int_{A_{k}^{z}}|x|^{-\hat{q} \alpha_{1}}\left|\left(|x|^{\alpha_{1}}|u|-k\right)_{+}\right|^{\hat{q}} d x\right)^{\frac{\hat{q}(1+\chi)}{(1)}}\left(\int_{A_{k}^{z}} d x\right)^{\frac{\chi}{1+\chi} \frac{\hat{r}}{\hat{q}}} d z\right]^{\frac{2(1+\chi)}{\hat{r}}} \\
& \leq C_{f}\left[\int_{0}^{t_{1}}\left(\int_{A_{k}^{z}}|x|^{-\hat{q} \alpha_{1}}\left|\left(|x|^{\alpha_{1}}|u|-k\right)_{+}\right|^{\hat{q}} d x\right)^{\frac{\hat{r}}{\hat{q}}} d z\right]^{\frac{2}{\hat{r}}}\left[\int_{0}^{t_{1}}\left(\int_{A_{k}^{z}} d x\right)^{\frac{\hat{r}}{\hat{q}}} d z\right]^{\frac{2 \chi}{\hat{r}}} \\
& \leq C_{1} C_{f}\left\|\left(|x|^{\alpha_{1}}|u|-k\right)_{+}\right\|_{L^{\hat{r}}\left(0, t_{1} ; L_{\alpha_{1}} \hat{q}_{1}\left(A_{k}^{z}\right)\right)}^{2} \cdot\left(\int_{0}^{t_{1}}\left(\int_{A_{k}^{z}}|x|^{-\alpha_{1} \hat{q}} d x\right)^{\frac{\hat{r}}{\hat{q}}} d z\right)^{\frac{2 \chi}{\hat{r}}} \\
& =C_{1} C_{f}\left\|\left(|x|^{\alpha_{1}}|u|-k\right)_{+}\right\|_{L^{\hat{r}}\left(0, t_{1} ; L_{\alpha_{1}}^{\hat{q}}\left(A_{k}^{z}\right)\right)}^{2} \cdot \mu(k)^{\frac{2 \chi}{\hat{r}}} .
\end{aligned}
$$

Applying Proposition 2.4 with $\theta=\frac{N}{2}-\frac{N}{\hat{q}}$, we have

$$
\begin{aligned}
\int_{0}^{t_{1}} & \int_{A_{k}^{z}}|x|^{-2 \alpha_{1}}|f|\left(|x|^{\alpha_{1}}|u|-k\right)_{+}^{2} d x d z \\
\leq & C_{2} C_{f} \mu(k)^{\frac{2 \chi}{\hat{r}}}\left(\int_{0}^{t_{1}}\left\|\nabla\left[\left(|x|^{\alpha_{1}}|u|-k\right)_{+} \operatorname{sign}(u)\right]\right\|_{L_{\alpha_{1}}^{2} \theta}^{\hat{r} \theta} \cdot\left\|\left(|x|^{\alpha_{1}}|u|-k\right)_{+}\right\|_{L_{\alpha_{1}}^{2}}^{(1-\theta) \hat{r}} d z\right)^{\frac{2}{\hat{r}}} \\
\leq & C_{2} C_{f} \mu(k)^{\frac{2 \chi}{\hat{r}}}\left\|\left(|x|^{\alpha_{1}}|u|-k\right)_{+}\right\|_{L^{\infty}\left(0, t_{1} ; L_{\alpha_{1}}^{2}\left(A_{k}^{z}\right)\right)}^{2(1-\theta)} \\
& \cdot\left(\int_{0}^{t_{1}}\left\|\nabla\left[\left(|x|^{\alpha_{1}}|u|-k\right)_{+} \operatorname{sign}(u)\right]\right\|_{L_{\alpha_{1}}^{2} \theta}^{\hat{r}^{2}} d z\right)^{\frac{2}{\hat{r}}} .
\end{aligned}
$$

Thus, applying Young's inequality, we get

$$
\begin{aligned}
& \int_{0}^{t_{1}} \int_{A_{k}^{z}}|x|^{-2 \alpha_{1}}|f|\left(|x|^{\alpha_{1}}|u|-k\right)_{+}^{2} d x d z \\
& \leq C_{2} C_{f} \mu(k)^{\frac{2 \chi}{\hat{r}}}\left\|\left(|x|^{\alpha_{1}}|u|-k\right)_{+}\right\|_{L^{\infty}\left(0, t_{1} ; L_{\alpha_{1}}^{2}(\Omega)\right)}^{2} \\
& \quad+C_{2} C_{f} \mu(k)^{\frac{2 \chi}{\hat{r}}} \theta\left(\int_{0}^{t_{1}}\left\|\nabla\left[\left(|x|^{\alpha_{1}}|u|-k\right)_{+} \operatorname{sign}(u)\right]\right\|_{L_{\alpha_{1}}^{2} \theta}^{\hat{r}_{2}} d z\right)^{\frac{2}{\hat{r} \theta}} .
\end{aligned}
$$


Since $\hat{r}$ and $\hat{q}$ satisfy (5.8), then $\hat{r} \theta=2$ and we obtain,

$$
\begin{aligned}
& \int_{0}^{t_{1}} \int_{A_{k}^{z}}|x|^{-2 \alpha_{1}}|f|\left(|x|^{\alpha_{1}}|u|-k\right)_{+}^{2} d x d z \\
& \leq C_{2} C_{f} \mu(k)^{\frac{2 \chi}{r}}\left[\left\|\left(|x|^{\alpha_{1}}|u|-k\right)_{+}\right\|_{L^{\infty}\left(0, t_{1} ; L_{\alpha_{1}}^{2}(\Omega)\right)}^{2}\right. \\
& \left.\quad+\left\|\nabla\left[\left(|x|^{\alpha_{1}}|u|-k\right)_{+} \operatorname{sign}(u)\right]\right\|_{L^{2}\left(0, t_{1} ; L_{\alpha_{1}}^{2}(\Omega)\right)}^{2}\right] .
\end{aligned}
$$

On the other hand, the second term on the right hand in (5.7) satisfies

$$
\begin{aligned}
\int_{0}^{t_{1}} \int_{A_{k}^{z}}|f| d x d z & =\int_{0}^{t_{1}} \int_{A_{k}^{z}}|x|^{2 \alpha(1+\chi)}|x|^{-2 \alpha(1+\chi)}|f| d x d z \\
& \leq C_{3}\left(\int_{0}^{t_{1}}\left(\int_{A_{k}^{z}}|f|^{q} d x\right)^{\frac{r}{q}} d z\right)^{\frac{1}{r}} \cdot\left(\int_{0}^{t_{1}}\left(\int_{A_{k}^{z}}|x|^{-\alpha \hat{q}} d x\right)^{\frac{\hat{r}}{\hat{q}}} d z\right)^{\frac{1}{r^{\prime}}} \\
& =C_{3} C_{f} \mu(k)^{\frac{2(1+\chi)}{\hat{r}}} .
\end{aligned}
$$

Denoting

$$
\begin{aligned}
\left|\left(|x|^{\alpha_{1}}|u|-k\right)_{+}\right|_{A_{k}^{t}}^{2}= & \left\|\left(|x|^{\alpha_{1}}|u|-k\right)_{+}\right\|_{L^{\infty}\left(0, t ; L_{\alpha}^{2}(\Omega)\right)}^{2} \\
& +\left\|\nabla\left[\left(|x|^{\alpha_{1}}|u|-k\right)_{+} \operatorname{sign}(u)\right]\right\|_{L^{2}\left(0, t ; L_{\alpha}^{2}(\Omega)\right)}^{2}
\end{aligned}
$$

from (5.6), we conclude that

$$
C\left|\left(|x|^{\alpha_{1}}|u|-k\right)_{+}\right|_{A_{k}^{t_{1}}}^{2} \leq C_{2} C_{f}\left|\left(|x|^{\alpha_{1}}|u|-k\right)_{+}\right|_{A_{k}^{t_{1}}}^{2} \cdot \mu(k)^{\frac{2 \chi}{\hat{r}}}+C_{3} C_{f} \mu(k)^{\frac{2(1+\chi)}{\hat{r}}} .
$$

Let us choose $t_{1}$ small enough such that

$$
C_{2} C_{f} t_{1}^{\frac{2 \chi}{\hat{r}}}\left\||x|^{-\alpha_{1}}\right\|_{L^{\hat{q}}(\Omega)}^{\frac{2 \chi}{\hat{q}}}<C .
$$

Thus again by Proposition 2.4, we deduce that there exist $C_{4}$ and $C_{5}$ satisfying

$$
C_{5}\left\|\left(|x|^{\alpha_{1}}|u|-k\right)_{+}\right\|_{L^{\hat{r}}\left(0, t_{1} ; L_{\alpha}^{\hat{q}}(\Omega)\right)}^{2} \leq C_{4}\left|\left(|x|^{\alpha_{1}}|u|-k\right)_{+}\right|_{A_{k}^{t_{1}}}^{2} \leq C_{f} \mu(k)^{\frac{2(1+\chi)}{\hat{r}}} .
$$

Consider $h>k>0$, hence it results $A_{h} \subset A_{k}$, where $A_{h}$ is as in (5.1), and

$$
\mu(h) \leq \frac{C_{6}}{(h-k)^{\hat{r}}} \mu(k)^{1+\chi} .
$$

Applying Lemma 2.7, we conclude that there exists a constant $d$, depending only on $q, r,\|f\|_{L^{r}\left(0, T ; L^{q}(\Omega)\right)}$ and $\alpha_{1}$, such that $\mu(d)=0$, that is

$$
\left\||x|^{\alpha_{1}} u\right\|_{L^{\infty}\left(\Omega \times\left[0, t_{1}\right]\right)} \leq d .
$$

Iterating this procedure in the sets $\Omega \times\left[t_{1}, 2 t_{1}\right], \ldots, \Omega \times\left[j t_{1}, T\right]$, where $T-j t_{1} \leq$ $t_{1}$, (notice that the process works since in all these sets (5.9) is verified), we can conclude that $L^{\infty}$ bound holds true in $Q$. To conclude, notice that by the previous proof we also deduce that $\left(|x|^{\alpha_{1}}|u|-k\right)_{+} \operatorname{sign}(u) \in V_{\alpha_{1}}^{2}(Q)$. 


\subsection{Proof of Theorem 4.3}

For every $n \in \mathbf{N}$, let us consider $u_{n} \in V^{2}(Q) \cap L^{\infty}(Q)$, the solution to the approximated problem

$$
\begin{cases}u_{n t}-\Delta u_{n}=\lambda \frac{u_{n}}{|x|^{2}+\frac{1}{n}}+T_{n}(f) & \text { in } \Omega \times(0, T), \\ u_{n}(x, t)=0 \quad \text { on } \partial \Omega \times(0, T), & \\ u_{n}(x, 0)=0 \quad \text { in } \Omega, & \end{cases}
$$

where $T_{n}$ is as in (3.2). Reasoning as in the proof of Theorem 1.3 the assertion follows showing that the sequence $\left[\left(1+|x|^{\alpha_{1}}\left|u_{n}\right|^{\gamma}-1\right] \operatorname{sign}\left(u_{n}\right)\right.$ is equibounded in $V_{\alpha_{1}}^{2}(Q) \cap L^{\delta}\left(0, T ; L_{\alpha_{1}}^{m}(\Omega)\right)$. To this aim we start proving the following estimate.

Lemma 5.1. There exists a positive constant $C_{0}$, depending only on $\Omega$ and $\|f\|_{L^{1}(Q)}$, thus independent of $n$, such that

$$
\left\||x|^{-\alpha_{1}} u_{n}\right\|_{L^{\infty}\left(0, T ; L^{1}(\Omega)\right)} \leq C_{0} .
$$

Proof of Lemma 5.1. Let us define

$$
\psi_{1}(s)=\int_{0}^{s} T_{1}(\sigma) d \sigma
$$

Notice that it results

$$
|s|-\frac{1}{2} \leq \psi_{1}(s) \leq|s|, \quad \forall s \in \mathbf{R} .
$$

Hence taking $|x|^{-\alpha_{1}} T_{1}\left(u_{n}\right)$ as a test function in (5.10), using the previous inequality and the definition of $\alpha_{1}$ we obtain that (5.11) holds true.

Using the previous lemma, we can now prove the thesis of Theorem 4.3. Define for $s \in \mathbf{R}$

$$
\Psi(s)=\int_{0}^{s}|x|^{-\alpha_{1}}\left[\left(1+|x|^{\alpha_{1}}|y|\right)^{2 \gamma-1}-1\right] d y,
$$

where $\gamma$ is a positive constant satisfying $\gamma>\frac{1}{2}$ that will be chosen later as in the statement of the Theorem 4.3. It results

$$
\Psi(s) \geq|x|^{-2 \alpha_{1}} \frac{\left(1+|x|^{\alpha_{1}}|s|\right)^{2 \gamma}-1}{2 \gamma}-|x|^{-\alpha_{1}}|s|, \quad \forall s \in \mathbf{R} .
$$

Choose $v_{n}=|x|^{-\alpha_{1}}\left[\left(1+|x|^{\alpha_{1}}\left|u_{n}\right|\right)^{2 \gamma-1}-1\right] \operatorname{sign}\left(u_{n}\right)$ as a test function in (5.10). Recalling that $u_{n}(x, 0)=0$, we obtain

$$
\begin{gathered}
\int_{\Omega} \Psi\left(u_{n}(x, t)\right) d x+\int_{0}^{t} \int_{\Omega} \nabla u_{n} \nabla\left\{|x|^{-\alpha_{1}}\left[\left(1+|x|^{\alpha_{1}}\left|u_{n}\right|\right)^{2 \gamma-1}-1\right] \operatorname{sign}\left(u_{n}\right)\right\} d x d \tau \\
\leq \lambda \int_{0}^{t_{1}} \int_{\Omega} \frac{\left|u_{n}\right|}{|x|^{\alpha_{1}+2}}\left[\left(1+|x|^{\alpha_{1}}\left|u_{n}\right|\right)^{2 \gamma-1}-1\right] d x d \tau+\int_{0}^{t} \int_{\Omega}|f|\left|v_{n}\right| d x d \tau
\end{gathered}
$$

where $0<t \leq T$. 
Thanks to the divergence theorem, we have that

$$
\begin{aligned}
& \int_{0}^{t_{1}} \quad \int_{\Omega}\left[\nabla u_{n} \nabla|x|^{-\alpha_{1}}\right]\left[\left(1+|x|^{\alpha_{1}}\left|u_{n}\right|\right)^{2 \gamma-1}-1\right] \operatorname{sign}\left(u_{n}\right) d x d t \\
& \quad+\int_{0}^{t_{1}} \int_{\Omega}\left(\nabla|x|^{-\alpha_{1}}\right) u_{n} \nabla\left[\left(\left(1+|x|^{\alpha_{1}}\left|u_{n}\right|\right)^{2 \gamma-1}-1\right) \operatorname{sign}\left(u_{n}\right)\right] d x d t \\
& =\lambda \int_{0}^{t_{1}} \int_{\Omega} \frac{\left|u_{n}\right|}{|x|^{\alpha_{1}+2}}\left[\left(1+|x|^{\alpha_{1}}\left|u_{n}\right|\right)^{2 \gamma-1}-1\right] d x d t
\end{aligned}
$$

Since $|x|^{-2 \alpha_{1}}\left(\nabla|x|^{\alpha_{1}} u_{n}\right)=|x|^{-\alpha_{1}} \nabla u_{n}-u_{n} \nabla|x|^{-\alpha_{1}}$, we get

$$
\begin{aligned}
& \int_{\Omega} \Psi\left(u_{n}(x, t)\right) d x+\int_{0}^{t} \int_{\Omega}|x|^{-2 \alpha_{1}} \nabla\left(|x|^{\alpha_{1}} u_{n}\right) \nabla\left[\left\{\left(1+|x|^{\alpha_{1}}\left|u_{n}\right|\right)^{2 \gamma-1}-1\right\} \operatorname{sign}\left(u_{n}\right)\right] d x d \tau \\
& \quad \leq \int_{0}^{t} \int_{\Omega}|f|\left|v_{n}\right| d x d \tau
\end{aligned}
$$

where $0<t \leq T$. Therefore, it results

$$
\begin{aligned}
& \int_{\Omega} \Psi(u(x, t)) d x+\left.\left.(2 \gamma-1) \int_{0}^{t} \int_{\Omega}|x|^{-2 \alpha_{1}}|\nabla| x\right|^{\alpha_{1}} u_{n}\right|^{2}\left(1+|x|^{\alpha_{1}}\left|u_{n}\right|\right)^{(2 \gamma-2)} d x d \tau \\
& \quad \leq \int_{0}^{t} \int_{\Omega}|f|\left|v_{n}\right| d x d \tau .
\end{aligned}
$$

Applying Hölder's inequality, (5.13) and Lemma 5.1, we get

$$
\begin{aligned}
& \frac{1}{2 \gamma} \sup _{[0, T]} \int_{\Omega}|x|^{-2 \alpha_{1}}\left(1+|x|^{\alpha_{1}}\left|u_{n}\right|\right)^{2 \gamma} d x \\
& \quad+\frac{(2 \gamma-1)}{\gamma^{2}} \int_{0}^{T} \int_{\Omega}|x|^{-2 \alpha_{1}}\left|\nabla\left\{\left[\left(1+|x|^{\alpha_{1}}\left|u_{n}\right|\right)^{\gamma}-1\right] \operatorname{sign}\left(u_{n}\right)\right\}\right|^{2} d x d t \\
& \leq|||x|^{-h} f \|_{L^{r}\left(0, T ; L^{q}(\Omega)\right)}\left(\int_{0}^{T}\left[\int_{\Omega}|x|^{\left(h-\alpha_{1}\right) q^{\prime}}\left(1+|x|^{\alpha_{1}}\left|u_{n}\right|\right)^{(2 \gamma-1) q^{\prime}} d x\right]^{\frac{r^{\prime}}{q^{\prime}}} d t\right)^{\frac{1}{r^{\prime}}} \\
& \quad+\frac{1}{2 \gamma}|\Omega|+\left|\left\|\left.x\right|^{-\alpha_{1}} u_{n}\right\|_{L^{\infty}\left(0, T ; L^{1}(\Omega)\right)}=C_{1}+C_{f}\right. \\
& \quad \times\left(\int_{0}^{T}\left|\left\|\left.x\right|^{\left(h-\alpha_{1}\right)}\left(1+|x|^{\alpha_{1}}\left|u_{n}\right|\right)^{(2 \gamma-1)}\right\|_{L^{q^{\prime}}(\Omega)}^{r^{\prime}} d t\right)^{\frac{1}{r^{\prime}}},\right.
\end{aligned}
$$

where $C_{1}=\frac{|\Omega|}{2 \gamma}+C_{0}$, with $C_{0}$ as in (5.11) and $C_{f}=\left\||x|^{-h} f\right\|_{L^{r}\left(0, T ; L^{q}(\Omega)\right)}$. Denoting by

$$
A=\left(\int_{0}^{T}\left\||x|^{\left(h-\alpha_{1}\right)}\left(1+|x|^{\alpha_{1}}\left|u_{n}\right|\right)^{(2 \gamma-1)}\right\|_{L^{q^{\prime}}(\Omega)}^{r^{\prime}} d t\right)^{\frac{1}{r^{\prime}}}
$$

we have

$$
\sup _{[0, T]} \int_{\Omega}|x|^{-2 \alpha_{1}}\left(1+|x|^{\alpha_{1}}\left|u_{n}\right|\right)^{2 \gamma} d x \leq 2 \gamma\left(C_{1}+C_{f} A\right) .
$$


Thanks to Sobolev's weight inequality, we get

$$
\begin{aligned}
& \int_{0}^{T}\left[\int_{\Omega}\left[|x|^{-\alpha_{1}}\left(\left(1+|x|^{\alpha_{1}}\left|u_{n}\right|\right)^{\gamma}-1\right)\right]^{2^{*}} d x\right]^{\frac{2}{2^{*}}} d t \\
& \quad \leq C_{S o b}^{2}\left(\frac{(2 \gamma-1)}{\gamma^{2}}\right)^{-1}\left(C_{1}+C_{f} A\right) .
\end{aligned}
$$

Moreover it is easy to prove the existence of two positive constants $C_{2}$ and $C_{3}$, independent on $k$, (for example $C_{2}=C_{3}=2^{2^{*}}$ ), such that

$$
\left[|x|^{-\alpha_{1}}\left(\left(1+|x|^{\alpha_{1}}\left|u_{n}\right|\right)^{\gamma}-1\right)\right]^{2^{*}} \geq C_{2}|x|^{-2^{*} \alpha_{1}}\left(1+|x|^{\alpha_{1}}\left|u_{n}\right|\right)^{2^{*} \gamma}-C_{3} .
$$

Hence we can conclude that

$$
\int_{0}^{T}\left[\int_{\Omega}|x|^{-2^{*} \alpha_{1}}\left(1+|x|^{\alpha_{1}}\left|u_{n}\right|\right)^{2^{*} \gamma} d x\right]^{\frac{2}{2^{*}}} d t \leq C_{4}+C_{5} A
$$

where $C_{4}=\left(\frac{2}{C_{2}}\right)^{\frac{2}{2^{*}}} C_{S o b}^{2}\left[\frac{(2 \gamma-1) \alpha}{\gamma^{2}}\right]^{-1} C_{1}+\left(\frac{2 C_{3}}{C_{2}}\right)^{\frac{2}{2^{*}}} T$, and $C_{5}=\left(\frac{2}{C_{2}}\right)^{\frac{2}{2^{*}}}$ $C_{S o b}^{2}\left[\frac{(2 \gamma-1) \alpha}{\gamma^{2}}\right]^{-1} C_{f}$. We proceed analogously to the proof of Theorem 1.3 distinguishing now two cases depending on the size of $q$.

First case: $1<q<\frac{N r}{N+2(r-1)}$, that is the zone E in Fig. 1. In this case (3.19) holds true and hence, we can apply Hölder's inequality to estimate $A$ and we get

$$
A \leq T^{\frac{1}{r^{\prime}}-\frac{2^{*}}{2 q^{\prime}}}\left[\int_{0}^{T}\left(\int_{\Omega}|x|^{\left(h-\alpha_{1}\right) q^{\prime}}\left(1+|x|^{\alpha_{1}}\left|u_{n}\right|\right)^{(2 \gamma-1) q^{\prime}} d x\right)^{\frac{2}{2^{*}}} d t\right]^{\frac{2^{*}}{2 q^{\prime}}}
$$

Thus from (5.17), we deduce that

$$
\begin{aligned}
& \int_{0}^{T}\left[\int_{\Omega}\left[|x|^{-2^{*} \alpha_{1}}\left(1+|x|^{\alpha_{1}}\left|u_{n}\right|\right)^{2^{*} \gamma}\right] d x\right]^{\frac{2}{2^{*}}} d t \\
& \quad \leq C_{4}+C_{6}\left[\int_{0}^{T}\left(\int_{\Omega}|x|^{\left(h-\alpha_{1}\right) q^{\prime}}\left(1+|x|^{\alpha_{1}}\left|u_{n}\right|\right)^{(2 \gamma-1) q^{\prime}} d x\right)^{\frac{2}{2^{*}}} d t\right]^{\frac{2^{*}}{2 q^{\prime}}},
\end{aligned}
$$

where $C_{6}=C_{5} T^{\frac{1}{r^{\prime}}-\frac{2^{*}}{2 q^{\prime}}}$. We choose $2^{*} \gamma=(2 \gamma-1) q^{\prime}$, that is, $\gamma=\gamma_{1}=\frac{1}{2} \cdot \frac{q(N-2)}{(N-2 q)}$ and $\left(h-\alpha_{1}\right) q^{\prime}=-2^{*} \alpha_{1}$, namely, $h=\alpha_{1} \frac{2 N-q(N+2)}{q(N-2)}$. Notice that since $q<\frac{N}{2}$ it results $\frac{2^{*}}{2 q^{\prime}}<1$ and thus we get

$$
\int_{0}^{T}\left(\int_{\Omega}|x|^{\left(h-\alpha_{1}\right) q^{\prime}}\left(1+|x|^{\alpha_{1}}\left|u_{n}\right|\right)^{\left(2 \gamma_{1}-1\right) q^{\prime}} d x\right)^{\frac{2}{2^{*}}} d t \leq C_{7},
$$

where $C_{7}$ depends only on $C_{4}, C_{6}, N, 2^{*} \gamma_{1}=q^{* *}$ and $q$. As obviously, it results

$$
\left(1+|x|^{\alpha_{1}}\left|u_{n}\right|\right)^{2 \gamma} \geq\left|\left(1+|x|^{\alpha_{1}}\left|u_{n}\right|\right)^{\gamma}-1\right|^{2},
$$


using (5.20) and (5.18) in (5.15), since $2^{*}\left(\gamma_{1}-1\right) \alpha_{1}=-h q^{* *}$ and $2^{*} \gamma_{1}=q^{* *}$ by $(5.20)$ the assert follows. In particular, we deduce

$$
\left\||x|^{-h} u\right\|_{L^{p}\left(0, T ; L^{q^{* *}}(\Omega)\right)} \leq C_{8},
$$

with $p=\frac{q(N-2)}{(N-2 q)}>q$ and $C_{8}=C_{7}^{\frac{1}{q^{* *}}}$.

Second case: $\frac{N r}{N+2(r-1)} \leq q<\frac{N}{2} r^{\prime}$, that is zone C in Fig. 1 .

In this case we will choose $\gamma$ satisfying (3.28) and we define $\theta \in(0,1)$ as in (3.29). Then it follows

$$
\begin{aligned}
& \left\||x|^{\frac{\left(h-\alpha_{1}\right)}{(2 \gamma-1)}}\left(1+|x|^{\alpha_{1}}\left|u_{n}\right|\right)\right\|_{L^{(2 \gamma-1) q^{\prime}}}^{r^{\prime}(2 \gamma-1)} \\
& \leq\left|\left\||x|^{\frac{\left(h-\alpha_{1}\right)}{(2 \gamma-1)}}\left(1+|x|^{\alpha_{1}}\left|u_{n}\right|\right)\right\|_{L^{2 \gamma}}^{(1-\theta) r^{\prime}(2 \gamma-1)}\left\|\left(|x|^{\frac{\left(h-\alpha_{1}\right)}{(2 \gamma-1)}}\left(1+|x|^{\alpha_{1}}\left|u_{n}\right|\right)\right)\right\|_{L^{2^{*} \gamma}}^{\theta r^{\prime}(2 \gamma-1)}\right. \\
& \leq\left.|| x\right|^{\frac{\left(h-\alpha_{1}\right)}{(2 \gamma-1)}}\left(1+|x|^{\alpha_{1}}\left|u_{n}\right|\right) \|_{L^{\infty}\left(0, T ; r^{\prime}(2 \gamma(\Omega))\right.}^{(1-\theta)} \\
& \quad \times\left(\int_{\Omega}|x|^{\frac{\left(h-\alpha_{1}\right)}{(2 \gamma-1)}}\left(1+|x|^{\alpha_{1}}\left|u_{n}\right|\right)^{2^{*} \gamma} d x\right)^{\frac{\theta r^{\prime}(2 \gamma-1)}{2^{*} \gamma}} .
\end{aligned}
$$

Integrating on time we obtain

$$
\begin{aligned}
A^{r^{\prime}}= & \int_{0}^{T}\left\||x|^{\frac{\left(h-\alpha_{1}\right)}{(2 \gamma-1)}}\left(1+|x|^{\alpha_{1}}\left|u_{n}\right|\right)\right\|_{L^{(2 \gamma-1) q^{\prime}}}^{r^{\prime}(2 \gamma-1)} d t \\
\leq & \left\||x|^{\frac{\left(h-\alpha_{1}\right)}{(2 \gamma-1)}}\left(1+|x|^{\alpha_{1}}\left|u_{n}\right|\right)\right\|_{L^{\infty}\left(0, T ; L^{2 \gamma}(\Omega)\right)}^{2 \gamma \mu_{1}} \\
& \times \int_{0}^{T}\left(\int_{\Omega}|x|^{\frac{\left(h-\alpha_{1}\right)}{(2 \gamma-1)} 2^{*} \gamma}\left(1+|x|^{\alpha_{1}}\left|u_{n}\right|\right)^{2^{*} \gamma} d x\right)^{\frac{2}{2^{*} \mu_{2}}} d t
\end{aligned}
$$

with $\mu_{1}$ and $\mu_{2}$ as in (3.31). The proof now is similar at all to that of Theorem 1.3. Again we need that (3.32) holds true and since from (3.29) we know that (3.33) holds, the condition (3.32) becomes (3.34). Since $N \geq 3$, (3.35) is satisfied. Thus since we are assuming $q<\frac{N}{2} r^{\prime},(3.34)$ is satisfied if (and only if) (3.36) holds true. With such a choice we get

$$
\begin{aligned}
A^{r^{\prime}}= & \int_{0}^{T}\left\||x|^{\frac{\left(h-\alpha_{1}\right)}{(2 \gamma-1)}}\left(1+|x|^{\alpha_{1}}\left|u_{n}\right|\right)\right\|_{L^{(2 \gamma-1) q^{\prime}}}^{r^{\prime}(2 \gamma-1)} d t \\
\leq & C_{8}\left(\sup _{[0, T]} \int_{\Omega}|x|^{2 \gamma \frac{\left(h-\alpha_{1}\right)}{(2 \gamma-1)}}\left(1+|x|^{\alpha_{1}}\left|u_{n}\right|\right)^{2 \gamma} d x\right)^{\mu_{1}} \\
& \times\left(\int_{0}^{T}\left[\int_{\Omega}|x|^{2^{*} \gamma \frac{\left(h-\alpha_{1}\right)}{(2 \gamma-1)}}\left(1+|x|^{\alpha_{1}}\left|u_{n}\right|\right)^{2^{*} \gamma} d x\right]^{\frac{2}{2^{*}}} d t\right)^{\mu_{2}},
\end{aligned}
$$

with $\mu_{2} \leq 1$ and $C_{8}=T^{1-\mu_{2}}$. Choose $\frac{\left(h-\alpha_{1}\right) \gamma}{(2 \gamma-1)}=-\alpha_{1}$, namely, $h=\frac{\alpha_{1}(1-\gamma)}{\gamma}$. From estimates (5.16) and (5.17) and the previous one, we obtain

$$
A^{r^{\prime}} \leq C_{8}\left[2 \gamma\left(C_{1}+C_{f} A\right)\right]^{\mu_{1}}\left(C_{4}+C_{5} A\right)^{\mu_{2}} \leq C_{9}+C_{10} A^{\beta},
$$

with $\beta=\mu_{1}+\mu_{2}=r^{\prime} \frac{(2 \gamma-1)}{2 \gamma}<r^{\prime}$. Therefore it follows

$$
\int_{0}^{T}\left(\int_{\Omega}|x|^{\frac{-\alpha_{1}}{\gamma}(2 \gamma-1) q^{\prime}}\left(1+|x|^{\alpha_{1}}\left|u_{n}\right|\right)^{(2 \gamma-1) q^{\prime}} d x\right)^{\frac{r^{\prime}}{q^{\prime}}} d t \leq C_{11},
$$


with $\gamma$ satisfying (3.28) and (4.7). Since $(2 \gamma-1) q^{\prime} \alpha_{1}\left(1-\frac{1}{\gamma}\right)=-h(2 \gamma-1) q^{\prime}$, then the previous inequality implies

$$
\int_{0}^{T}\left(\int_{\Omega}|x|^{-h(2 \gamma-1) q^{\prime}}\left|u_{n}\right|^{(2 \gamma-1) q^{\prime}} d x\right)^{\frac{r^{\prime}}{q^{\prime}}} d t \leq C_{11} .
$$

Using (5.22) and proceeding as in the previous case, the assert follows as soon as we show that it is possible to choose $\gamma=\gamma_{2}$, where $\gamma_{2}$ is as in (4.7). To this aim, notice that condition (3.28) is equivalent to require

$$
\left\{\begin{array}{l}
2 \gamma \geq q \\
2 \gamma(N-2 q) \leq q(N-2)
\end{array}\right.
$$

Hence if $q \geq \frac{N}{2}$, condition (5.23) is satisfied for every choice of $\gamma \geq \frac{q}{2}$, while if $q<\frac{N}{2}$, condition (5.23) is verified just for $\frac{q}{2} \leq \gamma<\gamma_{1}=\frac{1}{2} \cdot \frac{q(N-2)}{N-2 q}$.

Therefore, if $q \geq \frac{N}{2}$, condition (5.23) together with restriction (4.7) gives that it is sufficient to choose, $\frac{q}{2} \leq \gamma \leq \gamma_{2}=\frac{1}{2} \cdot \frac{q r N}{N r-2 q(r-1)}$. If otherwise $q<\frac{N}{2}$, then condition (5.23) and (4.7) are satisfied when $\frac{q}{2} \leq \gamma \leq \min \left\{\gamma_{1}, \gamma_{2}\right\}$. Since $\gamma_{2} \leq \gamma_{1}$ if and only if $q \geq \frac{N r}{N+2(r-1)}$, and $\gamma_{2} \geq \frac{q}{2}$, we have that it is sufficient to choose $\gamma=\gamma_{2}$ in order to satisfy conditions (3.28) and (4.7). Notice that it results

$$
\begin{aligned}
& \left(2 \gamma_{2}-1\right) r^{\prime}=\frac{r N(q-1)+2 q(r-1)}{r(N-2 q)+2 q} r^{\prime}, \\
& \left(2 \gamma_{2}-1\right) q^{\prime}=\frac{r N(q-1)+2 q(r-1)}{r(N-2 q)+2 q} q^{\prime} .
\end{aligned}
$$

Hence the assertion is proved.

Remark 5.2. Notice that for $\alpha_{1}=0$, namely, the Heat equation, it follows that $h=0$ and we have the regularity result in [14].

Observe that in the first case, that is, zones 4,5 , it follows that $h<0$ if and only if $q>\frac{2 N}{N+2}$, i.e, zone 4 . Therefore, in this case, it is sufficient to consider $\frac{f}{|x|^{|h|}} \in L^{r}\left(0, T ; L^{q}(\Omega)\right)$ and we get $\frac{u}{|x|^{|h|}} \in L^{p}\left(0, T ; L^{q^{* *}}(\Omega)\right)$.

Analogously in the second case, that is, zones $2,3,6$, it follows that $h<0$ if and only if $\gamma_{2}>1$ (zones 2,3). Therefore, in this case, it is sufficient to consider $\frac{f}{|x|^{|h|}} \in L^{r}\left(0, T ; L^{q}(\Omega)\right)$ and we get $\frac{u}{|x|^{|h|}} \in L^{2 \gamma_{2}}\left(0, T ; L^{2^{*} \gamma_{2}}(\Omega)\right)$.

Notice that for $q=\frac{2 N}{N+2}$ and $\gamma_{2}=1$, namely, in zones 2,3 , 4, it follows that for $f \in L^{r}\left(0, T ; L^{q}(\Omega)\right)$ and $\lambda \leq \Lambda_{N}$, then the solution $u$ to problem (1.5), satisfies $u \in V^{2}(Q)$.

Finally, notice that there is continuity in the results since $h_{1}=h_{2}$ on $q=\frac{N r}{N+2(r-1)}$.

Remark 5.3. As noticed in Remark 4.5, the results of Theorem 4.3 hold true also replacing the linear problem (4.1) with the nonlinear problem (4.3), where $\mathrm{g}$ is a Caratheodory function satisfying (4.4). As a matter of fact, in this case 
it is sufficient to replace the approximating problems (5.10) with

$$
\left\{\begin{array}{l}
u_{n t}-\Delta u_{n}=g_{n}\left(x, t, u_{n}, \nabla u_{n}\right)+T_{n}(f) \quad \text { in } \Omega \times(0, T), \\
u_{n}(x, t)=0 \quad \text { on } \partial \Omega \times(0, T), \\
u_{n}(x, 0)=0 \quad \text { in } \Omega
\end{array}\right.
$$

where $g_{n}$ is as in Remark 3.3 and to observe that all the estimates in the proof of Theorem 4.3 remain true since $g_{n}$ satisfies the inequality (3.49) in Remark 3.3.

\section{Acknowledgments}

The authors warmly thank the referees for helpful comments and suggestions.

\section{References}

[1] Abdellaoui, B., Colorado, E., Peral, I.: Existence and nonexistence results for a class of linear and semilinear parabolic equations related to some CaffarelliKohn-Nirenberg inequalities. J. Eur. Math. Soc. 6, 119-148 (2004)

[2] Abdellaoui, B., Peral, I.: Existence and non-existence results for quasilinear elliptic problems involving the p-Laplacian. Ann. Mat. Pura Appl. 182(3), 247$270(2003)$

[3] Abdellaoui, B., Peral, I.: Nonexistence results for quasilinear elliptic equations related to Caffarelli-Kohn-Niremberg inequality. Commun. Pure Appl. Anal. 2(4), 539-566 (2003)

[4] Abdellaoui, B., Peral, I., Primo, A.: Elliptic problems with a Hardy potential and critical growth in the gradient: non-resonance and blow-up results. J. Differ. Equ. 239(2), 386-416 (2007)

[5] Abdellaoui, B., Peral, I., Primo, A.: Breaking of resonance and regularizing effect of a first order quasi-linear term in some elliptic equations. Ann. I. H. Poincar. 25, 969-985 (2008)

[6] Aronson, D.G., Serrin, J.: Local behavior of solutions of quasilinear parabolic equations. Arch. Ration. Mech. Anal. 25, 81-122 (1967)

[7] Baras, P., Goldstein, J.: The heat equation with singular potential. Trans. Am. Math. Soc. 284, 121-139 (1984)

[8] Boccardo, L.: Hardy potentials and quasi-linear elliptic problems having natural growth terms. In: Progress in Nonlinear Differential equations and Their Applications, vol. 63, pp. 67-82. Birkhäuser, Boston (2005)

[9] Boccardo, L., Dall'Aglio, A., Gallouët, T., Orsina, L.: Nonlinear parabolic equations with measure data. J. Funct. Anal. 147(1), 237-258 (1997) 
[10] Boccardo, L., Dall'Aglio, A., Gallouët, T., Orsina, L.: Existence and regularity results for nonlinear parabolic equations. Adv. Math. Sci. Appl. 9, 1017-1031 (1999)

[11] Boccardo, L., Gallouet, T.: Nonlinear elliptic and parabolic equations involving measure data. J. Funct. Anal. 87, 149-169 (1989)

[12] Boccardo, L., Murat, F.: Almost everywhere convergence of the gradients of solutions to elliptic and parabolic equations. Nonlinear Anal. TMA 19(6), 581$597(1992)$

[13] Boccardo, L., Orsina, L., Peral, I.: A remark on existence and optimal summability of solutions of elliptic problems involving Hardy potential. Discrete Contin. Dyn. Syst. 16(3), 513 (2006)

[14] Boccardo, L., Porzio, M.M, Primo, A.: Summability and existence results for nonlinear parabolic equations. Nonlinear Anal. TMA 71, 978-990 (2009)

[15] Caffarelli, L., Kohn, R., Nirenberg, L.: First order interpolation inequalities with weights. Compos. Math. 53, 259-275 (1984)

[16] Chiarenza, F.M., Serapioni, R.P.: A Harnack inequality for degenerate parabolic equations. Commun. Partial Differ. Equ. 9(8), 719-749 (1984)

[17] DiBenedetto, E.: Degenerate Parabolic Equations. Springer, New York (1993)

[18] Garcia Azorero, J., Peral, I.: Hardy inequalities and some critical elliptic and parabolic problems. J. Differ. Equ. 144, 441-476 (1998)

[19] Gutiérrez, C.E., Wheeden, R.L.: Harnacks inequality for degenerate parabolic equations. Commun. Partial Differ. Equ. 16(4-5), 745-770 (1991)

[20] Ladyženskaja, O., Solonnikov, V.A., Ural'ceva, N.N.: Linear and quasilinear equations of parabolic type. Translations of the American Mathematical Society. American Mathematical Society, Providence (1968)

[21] Nirenberg, L.: On elliptic partial differential equations. Ann. Scuola Norm. Sup. Pisa 13 (1959)

[22] Porzio, M.M.: On the lack of the summability properties of solutions of elliptic problems with singular potentials. Preprint

[23] Porzio, M.M.: On some quasilinear elliptic equations involving Hardy potential. Rendiconti di matematica e delle sue applicazioni, Serie VII, vol. 27, fasc. III-IV, 277-299 (2007)

[24] Simon, J.: Compact sets in the space $L^{p}(0, T ; B)$. Ann. Mat. Pura Appl. 146, 65-96 (1987)

[25] Stampacchia, G.: Equations elliptiques du second ordre à coefficients discontinus. Seminaire de Mathematiques Superieures, 16. Les Presses de L'Universite de Montreal, Montreal (1966) 
Maria Michaela Porzio

Dipartimento di Matematica

Universita' di Roma 1, "La Sapienza"

Piazza A. Moro 2

00185 Rome

Italy

e-mail: porzio@mat.uniroma1.it

Ana Primo

ICMAT-CSIC, Ciudad Universitaria de Cantoblanco

28049 Madrid

Spain

e-mail: ana.primo@icmat.es

Received: 9 March 2011.

Accepted: 26 January 2012. 